\title{
Relativistic Celestial Mechanics with PPN Parameters
}

\author{
Sergei A. Klioner and Michael H. Soffel \\ Lohrmann Observatory, Dresden Technical University, \\ Mommsenstraße 13, D-01062 Dresden, Germany
}

(10 June 1999)

\begin{abstract}
Starting from the global parametrized post-Newtonian (PPN) reference system with two PPN parameters $\gamma$ and $\beta$ we consider a space-bounded subsystem of matter and construct a local reference system for that subsystem in which the influence of external masses reduces to tidal effects. Both the metric tensor of the local PPN reference system in the first post-Newtonian approximation as well as the coordinate transformations between the global PPN reference system and the local one are constructed in explicit form. The terms proportional to $\eta=4 \beta-\gamma-3$ reflecting a violation of the equivalence principle are discussed in detail.

We suggest an empirical definition of multipole moments which are intended to play the same role in PPN celestial mechanics as the BlanchetDamour moments in General Relativity. We also show that the tidal gravitational field as seen in the local PPN reference system can be expanded into powers of local coordinates similarly to the tidal expansion in General Relativity.

Starting with the metric tensor in the local PPN reference system we derive translational equations of motion of a test particle (an Earth satellite) in that system. The translational and rotational equations of motion for center of mass and spin of each of $N$ extended massive bodies possessing arbitrary
\end{abstract}


multipole structure are derived. All equations of motion are presented also in the form of multipole expansions. Several interesting features of the equations are discussed.

As an application of the general equations of motion a monopole-spin dipole model is considered and the known PPN equations of motion of mass monopoles with spins are rederived. For the first time, these equations are derived in a self-consistent manner which does not require any additional assumptions about the behavior of bodies such as secular stationarity.

PACS numbers: 04.25.Nx, 04.80.Cc, 95.10.Ce

Typeset using REVTEX 


\section{Contents}

\begin{tabular}{llr}
\hline$\quad$ Introduction & 5
\end{tabular}

$\begin{array}{lrr}\text { II } & \text { Notations } & 18\end{array}$

III Metric tensors and coordinate transformations 20

A The PPN metric tensor in the global reference system . . . . . . . . . . . 20

B $\quad$ Energy-momentum tensor for an ideal fluid . . . . . . . . . . . . . . . . . 23

C The PPN metric tensor in the local geocentric system . . . . . . . . . . . 25

D Coordinate transformations between the global and local reference systems 27

IV Matching of the global and local PPN metric tensors 27

A Matching of order $\mathcal{O}\left(c^{-2}\right)$ in $g_{00} \ldots \ldots \ldots$. . . . . . . . . . . . . . . . . 28

B Matching of order $\mathcal{O}\left(c^{-2}\right)$ in $g_{i j} \ldots \ldots \ldots \ldots$. . . . . . . . . . . . 29

C Matching of order $\mathcal{O}\left(c^{-3}\right)$ in $g_{0 i} \ldots \ldots \ldots$. . . . . . . . . . . 30

D Matching of order $\mathcal{O}\left(c^{-4}\right)$ in $g_{00} \ldots \ldots \ldots$. . . . . . . . . . . 32

E $\quad$ Energy-momentum tensor in the local reference system . . . . . . . . . . . 36

\begin{tabular}{|ll}
\hline Other forms of the local metrid & 37
\end{tabular}

\begin{tabular}{|lll}
\hline VI & Multipole expansions of the internal potentials & 39
\end{tabular}

VII Tidal expansions of the external potentials 41

VIII Translational equations of motion of a test particle in the local reference

$\begin{array}{ll}\text { system } & 44\end{array}$

A $\quad$ The equations of motion in closed form . . . . . . . . . . . . . . . . 44

B Multipole expansions of the equations of motion . . . . . . . . . . . . 45

IX Translational and rotational equations of motion of $N$ extended bodies 47

A Local equations of motion: $\mathcal{T}_{i \beta}^{0}=0 \ldots \ldots \ldots$. . . . . . . . . . 48 
B Local equations of motion: $\mathcal{T}_{: \beta}^{a p}=0$. . . . . . . . . . . . . . . . 50

C Local equations of motion: $\varepsilon_{a b c} X^{b} \mathcal{T}^{c p}{ }_{\beta}=0$. . . . . . . . . . . . . 51

D Multipole expansions of the relations between the Tolman and BD masses and mass dipoles . . . . . . . . . . . . . . . . . . . . . . . . 52

E Multipole-expanded local translational equations of motion . . . . . . . . 53

F Multipole-expanded rotational equations of motion . . . . . . . . . . . . . 55

G Mass monopole-spin dipole approximation . . . . . . . . . . . . . . . . . . 56

\begin{tabular}{|ll|}
\hline $\mathrm{X}$ & Outlook and conclusions \\
\hline
\end{tabular}

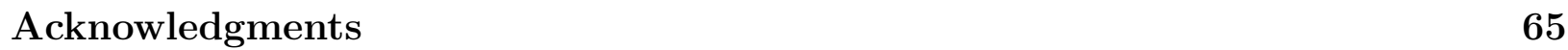

\begin{tabular}{ll}
\hline References & 67
\end{tabular} 


\section{INTRODUCTION}

Einstein's theory of gravity (the so-called General Theory of Relativity, GRT) in the last several decades has evolved far from being predominantly a playground for mathematicians. Not only that the field of experimental gravity has expanded and the number of experimental tests of GRT increased drastically, but certain results from GRT are taken for granted and implemented in software for solving technologically oriented problems. A good example is Very Long Baseline Interferometry (VLBI) that determines baselines on the surface of the Earth, and the position of the rotation pole with subcentimeter accuracy, the length of the day at the fraction of a millisecond level, and relative angles between two remote radio sources with a precision better than a milliarcsecond. In the VLBI analysis software relativistic effects like the gravitational time delay are routinely taken into account. Note that the gravitational light deflection amounts to $1.75^{\prime \prime}$ at the limb of the Sun and it decreases with the inverse impact parameter of the (unperturbed) light-ray from the Sun. This implies that the light deflection (more precisely the gravitational time delay) in the field of the Sun is measurable even for radio sources lying closely to $180^{\circ}$ from the Sun [1,2].

For the analysis of high precision optical astrometric measurements at the milliarcsecond level (e.g., HIPPARCOS) GRT became an indispensable tool [3]. For planned space interferometry with microarcsecond accuracies (DIVA, GAIA, SIM, FAME, etc.) relativity will play a crucial role and the whole process of observation (essentially, following the photons from the emission region onto the CCD-sensor) has to be formulated within the framework of Einstein's theory of gravity [4,5].

Astrometry is one important field of application of GRT, relativistic astrophysics (e.g., the physics of white dwarf stars, neutron stars, black holes or quasars, gravitational wave physics, cosmology) and celestial mechanics are further ones. Techniques used to gather information about the dynamics of astronomical bodies comprise the timing of millisecond pulsars at the sub-microsecond level, laser ranging to selected artificial satellites such as LAGEOS and to retro corner reflectors that have been placed on the lunar surface at the 
centimeter level and radar ranging to planets or spacecrafts (with, e.g., a few meters accuracy for the Viking landers on Mars).

Meanwhile it is well recognized that GRT enters every astronomical observation or measuring technique at a certain level of accuracy. Such a critical level has been reached for a variety of geodetic measurements including GPS and a long time ago already for the procedure to establish a terrestrial time scale (TT, TAI). In 1991 the International Astronomical Union and the International Union for Geodesy and Geophysics adopted recommendations implying the use of GRT in modeling of modern high-accuracy observations [6]. The International Earth Rotation Service responsible for precise monitoring of the Earth orientation parameters has also adopted relativistic models for several kinds of astronomical observations [7].

For practical applications one faces the problem of solving Einstein's field equations for complicated situations. Methods of numerical relativity might be employed in the future not only for problems related with gravity wave generation (e.g., for the description of coalescing binaries) or cosmology, but also for celestial mechanical problems in the solar system. However, the classical formulation of celestial mechanics is simplified considerably by the introduction of collective variables like mass-multipole moments, center of mass, etc. and one clearly wants to keep these advantages in a relativistic formulation. Note that the use of such collective variables is related with expansions in terms of a 'geometric coupling parameter' $\alpha \equiv L / R$, measuring the ratio between a characteristic linear dimension, $L$, of the bodies and a characteristic separation, $R$, between them.

Already soon after Einstein published his first papers on his theory of gravity, Einstein [8], Droste [9], De Sitter [10], and Lorentz and Droste [11] devised an approximation method (called "post-Newtonian") which allowed them to compare General Relativity with Newton's theory of gravity, and to predict several "relativistic effects" in celestial mechanics, such as the relativistic advance of the perihelion of planets, and the relativistic precession of the lunar orbit: this post-Newtonian approach to general relativistic celestial mechanics was subsequently developed by many authors, notably by Fock [12], Papapetrou [13], Chan- 
drasekhar and colleagues [14 16], Caporali [17], Grishchuk and Kopejkin [18] and many others (for a review of the development of the problem of motion in General Relativity see, e.g., Damour [19). Basically the post-Newtonian approach is a slow-motion and weak-field approximation to Einstein's theory of gravity. One introduces one or several dimensionless parameters for the $N$-body problem: $\beta_{e} \equiv v_{\text {orb }} / c$ (orbital velocities), $\beta_{i} \equiv v_{\text {int }} / c$ (internal or intrinsic rotational velocities), $\kappa_{e} \equiv G M /\left(c^{2} R\right)$ (external gravitational potential) and $\kappa_{i} \equiv G M /\left(c^{2} L\right)$ (internal gravitational potential) if $M$ is the mass of a body with dimension $L$ and $R$ is a typical distance to other bodies. Usually in the post-Newtonian approximation one assumes $\beta_{i}^{2}<\beta_{e}^{2} \sim \kappa_{e}<\kappa_{i}=\epsilon \ll 1$. E.g., $\epsilon<10^{-5}$ everywhere in the solar system.

All of the applications mentioned above require a correspondingly accurate relativistic theory of the gravitational $N$-body problem consisting of

(i) the external problem: to determine the motion of the centers of mass of the $N$ bodies and

(ii) the internal problem: to determine the motion of each body around its center of mass.

It is a remarkable fact of Newtonian celestial mechanics that these two sub-problems are coupled only very weakly. The first reason is the fact that the ellipticities (dimensionless mass-quadrupole moments or $J_{2}$-values) and higher mass-multipole moments of solar system bodies are all very small. Therefore the motions of the center of masses are dominated by the (approximately constant) masses of the bodies independent of internal motion, and the couplings of higher mass-multipole moments to higher-order derivatives of the external gravitational potential (i.e., couplings to the external curvature tensor in a geometric language) are very small. On the other hand the local environment of a body is influenced by external bodies only through the action of tidal forces that grow linearly with the distance from the body's center of mass and vary with $L$ according to $(R / L)^{3}$. This remarkable decoupling is sometimes expressed by the phrase "effacement of internal degrees of freedom in the global problem and of the external world in the local system". 
A second reason for this effacement is the principle of equivalence. In its weak form, applicable to Newtonian celestial mechanics, it asserts the equivalence of inertial mass with gravitational mass that has been tested by experiments with a relative precision of about $10^{-11}$ (see e.g., Will [1] for more detail).

As explained in great detail, e.g., in [20 22], the treatments of both sub-problems in the classical post-Newtonian approach are unsatisfactory. One usually considers the $N$-body problem in some asymptotically flat space-time that can be covered with one single global coordinate system, $x^{\mu} \equiv(c t, x, y, z) \equiv\left(c t, x^{i}\right), i=1,2,3$. In the classical formulation of postNewtonian theory concepts like "center of mass", "mass-multipole moments" (including the mass) or "mass-centered coordinates" are defined in this global coordinate system. E.g., for applications in the solar system the global coordinates usually will be barycentric ones and geocentric spatial coordinates classically were defined by $X^{i}=x^{i}-x_{E}^{i}(t)$, where $x_{E}^{i}(t)$ denotes the global coordinates of the "geocenter". Such definitions do not define a useful mass-centered frame in General Relativity, in the sense that it does not efface the external gravitational field down to tidal effects. In such a geocentric coordinate system relativistic effects are of order $\beta_{e}^{2} \sim 10^{-8}$ and not of order $\kappa_{i}=\left(G M_{E} / c^{2} R_{E}\right) \approx 7 \cdot 10^{-10}$ as one would expect. Clearly, the classical post-Newtonian theory tries to fit GRT into purely Newtonian framework and bears traces of Newton's absolute space and time.

In the recent decade the relativistic theory of astronomical reference systems in the framework of General Relativity has been considered in a detailed manner by a number of authors [23,24, 20,21,25,26,22, 27, 29]. These investigations were motivated both by theoretical interest to construct a proper reference system for a massive material subsystem and by practical requirements of astronomy 30 32 dictated by increased accuracy levels. The two most advanced and complete approaches to construct a physically adequate local reference system in General Relativity are the Brumberg-Kopeikin approach [20,21,25,26, 29. and the Damour-Soffel-Xu one [22,27,28, 33]. For the gravitational $N$-body problem both formalisms introduce a total of $N+1$ different coordinate systems: one set of global coordinates $x^{\mu}=\left(c t, x^{i}\right)$ and one set of local coordinates $X^{\alpha}=\left(c T, X^{a}\right)$ for each body comoving 
with the body under consideration. The relation between global and local (e.g., geocentric) coordinates describing one and the same event is assumed to be of the general form

$$
x^{\mu}\left(X^{\alpha}\right)=x_{\mathrm{E}}^{\mu}(T)+e_{a}^{\mu}(T) X^{a}+\xi^{\mu}(T, \mathbf{X}),
$$

where $\xi^{\mu}$ is at least quadratic in the local space coordinate $X^{a}$ and $x_{E}^{\mu}(T)$ describes the motion of a central worldline of the Earth (later chosen as the center of mass). Equivalently this transformation is described by

$$
X^{\alpha}\left(x^{\mu}\right)=X_{\mathrm{E}}^{\alpha}(t)+E_{i}^{\alpha}(t) r_{E}^{i}+\Xi^{\alpha}\left(t, \mathbf{r}_{E}\right),
$$

with $r_{E}^{i}(t)=x^{i}-x_{E}^{i}(t)$ and $\Xi^{\alpha}$ is assumed to be at least quadratic in $r_{E}^{i}$. Note that in passing from one form to the other one has to bear in mind that $x_{E}^{i}(T)$ and $X_{E}^{a}(t)$ describe two different points on the central geocentric worldline [25.33]. Suitable effacement conditions in the $X^{\alpha}$ system lead to constraints on the functions appearing in the coordinate transformations. Note, that these transformations involve space and time coordinates; they present generalized Lorentz transformations. Only by such transformations apparent Lorentz-contraction effects etc. can be avoided in the geocentric coordinate system.

Principle features and results of the theory of local reference system in the framework of General Relativity are:

- a description of the metric tensor $g_{\mu \nu}$ in the global system with only two gravitational potentials $\left(w, w^{i}\right)$ as functions of $x^{\mu}$; similarly, a description of $G_{\alpha \beta}$ in some local system with only two potentials $\left(W, W^{a}\right)$ [22];

- a new theory of astronomical reference frames, including the transformation rules for coordinates [20 22,27,29] and metric potentials [27];

- an improved description, with full post-Newtonian accuracy, of the gravitational structure of each body by means of a set of multipole moments, $\left(M_{L}^{A}, S_{L}^{A}\right)$, of a body $A$ (e.g., the Earth) which are linked in an operational way to what can be observed in the local gravitational environment of $A$ [22,22]; 
- a description of the influence of the external world in a local frame by means of some suitably defined tidal moments [20, 22, 27,29];

- translational [22,27] and rotational [28] equations of motion with full post-Newtonian accuracy and inclusion of all multipole moments;

- physical adequate equations of motion of a test particle (e.g., Earth satellite) in the local reference system 25, 33,29;

- physically adequate relativistic models for many kinds of observations (VLBI 34 36], high-accuracy positional observations [20,4], various methods of remote clock synchronization 37, etc.)

Despite the fact that GRT so far passed all experimental tests with flying colours (e.g., Misner et al. [38], Will [四]) people never lost interest in alternative theories of gravity. This is one reason why a parametrized post-Newtonian formalism (the PPN formalism) was developed to cover the post Newtonian limits of a whole class of metric theories of gravity including GRT. A primitive version of such a formalism was devised by Eddington [39] and Robertson [40] who introduced the space curvature parameter $\gamma$ and the nonlinearity parameter $\beta$ that are both equal to one $(\beta=\gamma=1)$ in GRT, but may take different values in alternative theories of gravity. Later Schiff [41 included terms from the rotation of bodies (gravito-magnetic terms). The classical PPN-formalism was developed mainly by Kenneth Nordtvedt in the late 60s and early 70s in collaboration with Clifford Will (Nordtvedt 42,43, Nordtvedt and Will [44, Will and Nordtvedt 454). More PPNparameters have been added to cover the post-Newtonian limits of a variety of scalar-tensor theories like the Brans-Dicke theory, vector tensor theories, bimetric theories, etc. The choice of PPN-parameters has changed over the years until some standard was achieved that can be found in Will [1]. Here, a total of ten PPN-parameters is introduced not only to distinguish between the various post-Newtonian limits of different theories of gravity, but also to provide a tool to characterize the precision of relevant gravitational experiments. In the following we will restrict ourselves to the Eddington-Robertson parameters $\beta$ and $\gamma$. The 
other PPN-parameters that are related with possible preferred locations, preferred frames or a violation of conservation of total momentum will not be considered here. This is not a crucial restriction for our approach that can be extended in a straightforward manner to any choice of PPN parameters. However, considering that the two parameters are the most important ones we confine ourselves to $\beta$ and $\gamma$ only.

This Eddington-Robertson-Schiff Nordtvedt-Will PPN-formalism is formulated in the spirit of classical post-Newtonian framework described above. Nevertheless, it was highly successful and lead to new predictions, e.g., related with a breakdown of the strong equivalence principle. Let us summarize briefly the most interesting results from the classical PPN-formalism that one would expect to be valid even in an improved version.

1. In every metric theory of gravity a test body of negligible extension and negligible gravitational self-energy will follow a geodesic in a suitably defined external metric $g_{\mu \nu}^{\text {ext }}$. From the usual form of the PPN metric one finds that the geodetic acceleration of a body $E$ is given by

$$
a_{E, \text { geodesic }}^{i}=-\sum_{B \neq E} G M_{B} \frac{r_{E B}^{i}}{r_{E B}^{3}}+\frac{1}{c^{2}}(\ldots)
$$

with suitably chosen masses $M_{B}$ of gravitating bodies and $r_{E B}^{i}=x_{E}^{i}-x_{B}^{i}$. Note that this acceleration (or the geodetic equation) follows from an action integral for the worldline of some central point of body $E$ of the form

$$
I_{\text {geodesic }}^{E}=-M_{E} c \int\left[-g_{\mu \nu}^{\text {ext }} d x^{\mu} d x^{\nu}\right]^{1 / 2}
$$

The corresponding $N$-body Lagrangian takes the form [1] $\left(\mathbf{r}_{A B}=\mathbf{x}_{A}-\mathbf{x}_{B}\right)$

$$
\begin{aligned}
\mathcal{L}_{\text {geodesic }}^{\mathrm{N} \text { body }}= & -\sum_{A} M_{A} c^{2}\left(1-\frac{1}{2} \frac{\mathbf{v}_{A}^{2}}{c^{2}}-\frac{1}{8} \frac{\mathbf{v}_{A}^{4}}{c^{4}}\right) \\
& +\frac{1}{2} \sum_{A} \sum_{B \neq A} \frac{G M_{A} M_{B}}{r_{A B}}\left[1+(2 \gamma+1) \frac{\mathbf{v}_{A}^{2}}{c^{2}}-(2 \beta-1) \sum_{C \neq A} \frac{G M_{C}}{c^{2} r_{A C}}\right. \\
& \left.-\frac{1}{2 c^{2}}(4 \gamma+3) \mathbf{v}_{A} \cdot \mathbf{v}_{B}-\frac{1}{2 c^{2}}\left(\mathbf{v}_{A} \cdot \mathbf{r}_{A B}\right)\left(\mathbf{v}_{B} \cdot \mathbf{r}_{A B}\right) \frac{1}{r_{A B}^{2}}\right] .
\end{aligned}
$$

2. In general a body $E$ with non negligible gravitational self-energy $\Omega_{E}$ will not follow a geodesic because of a violation of the Strong Equivalence Principle (e.g., Nordtvedt 42,43, 
Will [1]). In that case the value for the gravitational mass of $E, M_{E}^{(G)}$, will differ from that for the inertial mass $M_{E}$ by

$$
M_{E}^{(G)}=M_{E}+\eta \Omega_{E} / c^{2}
$$

Here, $\eta$ is the Nordtvedt-parameter. A classical result gives its relation to the EddingtonRobertson parameters

$$
\eta=4 \beta-\gamma-3
$$

This is the well-known Nordtvedt effect leading to a polarization of the lunar orbit around the Earth in the direction of the Sun if $\eta \neq 0$. The ratio of gravitational to inertial mass has an interesting and valuable interpretation. A violation of the Strong Equivalence Principle implies that in the local (e.g. geocentric) system the effects from the outside world cannot be effaced down to the usual tidal forces. Instead the "effective" gravitational constant, $G_{\text {local }}$, as experienced in the local $E$-frame becomes effectively space dependent

$$
G_{\text {local }}=G\left(1-\eta \frac{\bar{U}}{c^{2}}\right)
$$

Here, $\bar{U}$ is the external gravitational potential produced by the outside world, i.e. by all gravitating bodies different from $E$. Since second and higher order derivatives of $\bar{U}$ give rise to the usual tidal forces the effacement breaks down for the zeroth and first derivative of $\bar{U}$, taken at the geocenter $\mathbf{x}_{E}$. Considering the gravitational action between two points $\mathbf{x}$ and $\mathbf{x}^{\prime}$ one has effectively 46

$$
G_{\text {local }}=G\left[1-\eta \frac{\bar{U}\left(\mathbf{x}_{E}\right)}{c^{2}}-\frac{\eta}{2} \mathbf{a}_{E} \cdot\left(\mathbf{x}+\mathbf{x}^{\prime}\right)\right],
$$

where we have replaced $\nabla \bar{U}\left(\mathbf{x}_{E}\right)$ by the acceleration $\mathbf{a}_{E}$. Deriving the acceleration, e.g., of a satellite in the vicinity of the Earth by classical means, but with this expression for $G_{\text {local }}$ leads to the above ratio of gravitational to inertial mass of the Earth.

3. Already in Newtonian celestial mechanics extended bodies in general do not experience just the $1 / r^{2}$ acceleration when interacting with another body at distance $r$. Instead highermultipole mass moments $\left(C_{l m}, S_{l m}\right.$ or equivalently $M_{L}$ in the Cartesian language with $\left.l \geq 2\right)$ 
lead to a deviation of the 'geodetic' free-fall behavior. To lowest order this force of geodeticdeviation is given by

$$
a_{E, \text { quadrupole }}^{i}=\frac{1}{2 M_{E}} M_{j k}^{E} \partial_{i j k} \bar{U}\left(\mathbf{x}_{E}\right)=\frac{1}{2 M_{E}} I_{j k}^{E} \partial_{i j k} \bar{U}\left(\mathbf{x}_{E}\right)
$$

Here,

$$
I_{j k}^{E}=\int_{E} \rho^{*} r_{E}^{j} r_{E}^{k} d^{3} x
$$

is the Newtonian second-order mass tensor related with the Newtonian trace-free quadrupole tensor, $M_{j k}^{E}$, by

$$
M_{j k}^{E}=I_{j k}^{E}-\frac{1}{3} I_{s s}^{E} \delta_{j k}
$$

Note that the last equality in (1.10) follows from the Laplace equation in the absence of gravitational sources. Since second spatial derivatives of the external potential can be interpreted as components of a Newtonian external curvature tensor this quadrupole acceleration of body $E$ can be interpreted as resulting from a coupling of the body's second-moment mass tensor with the curvature tensor produced by the outside world. For that reason in any metric theory of gravity one expects the world line of a suitably chosen central point inside body $E$ to be influenced by the body's covariant second-moment mass tensor, $I_{\alpha \beta}^{E}$, via a coupling to the external curvature tensor $R_{\alpha \mu \beta \nu}^{\text {ext }}$ as described by the action integral (Nordtvedt 47])

$$
I=-M_{E} c \int\left[-\left(g_{\mu \nu}^{\mathrm{ext}}+\frac{I_{E}^{\alpha \beta}}{M_{E}} R_{\alpha \mu \beta \nu}^{\mathrm{ext}}\right) d x^{\mu} d x^{\nu}\right]^{1 / 2}
$$

In the Newtonian limit only the spatial components of $I_{E}^{\alpha \beta}$ (as measured in the local geocentric frame) contribute and from the action integral one derives an acceleration due to the second-moment mass tensor of

$$
a_{E, \text { quadrupole }}^{i}=\frac{c^{2}}{2 M_{E}} I_{j k}^{E} \partial_{i} R_{j 0 k 0}^{\mathrm{ext}}=\frac{1}{2 M_{E}} I_{j k}^{E} \partial_{i j k} \bar{U}\left(\mathbf{x}_{E}\right)
$$

As in Newton's theory, in GRT one finds that the trace of $I_{j k}^{E}$ does not lead to an additional acceleration because of the vacuum field equation (i.e., the vanishing of the Ricci-tensor in 
source-free regions). However, in general for $\gamma \neq 1$ the Ricci-tensor in vacuum does not vanish in alternative theories of gravity. In that case there will be an additional acceleration due to the extension-curvature coupling proportional to $\gamma-1$ even for spherical bodies with

$$
I_{j k}^{E}=\frac{1}{3} I^{E} \delta_{j k}
$$

and

$$
I^{E} \equiv \int_{E} \rho^{*} r_{E}^{i} r_{E}^{i} d^{3} x
$$

This implies that the effacement in GRT in the local (geocentric) system does not only lead to the ordinary value of the gravitational constant (since $\eta=0$ ), but also prevents extension-curvature couplings for spherical bodies (since $\gamma=1$ ).

4. Finally problems related with the spin (intrinsic angular momentum) have been treated extensively in the literature. There are two aspects that have to be tackled: (i) the influence of the spin upon the translational equation of motion and (ii) the motion of the spin vector itself (the rotational motion). Progress for solving the first problem has been achieved by Mathisson [48], Papapetrou [13], Corinaldesi and Papapetrou [49] without definitive conclusive results for GRT. However, in the first post-Newtonian approximation the situation is different. The spin-orbit and spin-spin coupling terms have been derived by Brumberg [50] who also discusses the physical implications in detail (see also Kalitzin [51] and Michalska [52]). They have also been derived using a one-graviton exchange theory by Barker et al. [53 and by Börner et al. [54 with parameters $\beta$ and $\gamma$ (see also Barker et al., [55 58]). Tulczyjew [59] has derived formally the same results using distributional sources. As first discussed by Damour [60], if we require invariance under Lorentz boosts, the spinorbit Lanrangian depends not only on positions and velocities but also on accelerations. Within the Lorentz-invariant Lagrangian formalism this is the manifestation of the wellknown Thomas precession [61]. The Lagrangian for the spin-orbit and spin-spin coupling terms for body $E$ takes the form $61,53,2]$ 


$$
\begin{aligned}
\mathcal{L}_{\text {spin }}^{E}= & +\frac{1}{c^{2}}(1+\gamma) G \sum_{B \neq E} \frac{\left(\left(\mathbf{v}_{E}-\mathbf{v}_{B}\right) \times \mathbf{r}_{E B}\right) \cdot\left(M_{E} \mathbf{S}_{B}+M_{B} \mathbf{S}_{E}\right)}{r_{E B}^{3}} \\
& +\frac{1}{2 c^{2}}\left(\mathbf{v}_{E} \times \mathbf{a}_{E}\right) \cdot \mathbf{S}_{E} \\
& -\frac{1}{2 c^{2}}(1+\gamma) G \sum_{B \neq E}\left[\frac{3\left(\mathbf{S}_{E} \cdot \mathbf{r}_{E B}\right)\left(\mathbf{S}_{B} \cdot \mathbf{r}_{E B}\right)}{r_{E B}^{5}}-\frac{\mathbf{S}_{E} \cdot \mathbf{S}_{B}}{r_{E B}^{3}}\right]
\end{aligned}
$$

Already to Newtonian order the motion of the spin is governed by extension-curvature couplings (precession of the equinoxes; e.g., Misner et al. 38]), were the quadrupole and higher order mass-multipole moments couple to the external curvature tensor. In the absence of such tidal forces the spin vector is Fermi-Walker transported (e.g., Misner et al. [38]) along the body's central world line. This Fermi-Walker transport has been extensively discussed in the literature. One finds that the spin-vector in the comoving frame precesses with respect to remote celestial objects (more precisely with respect to the local coordinate induced tetrad, see e.g. [1, 2]), i.e.

$$
\frac{d \mathbf{S}}{d \tau}=\mathbf{\Omega} \times \mathbf{S}
$$

with

$$
\boldsymbol{\Omega}=-\frac{1}{2} \frac{\mathbf{v} \times \mathbf{Q}}{c^{2}}-\frac{1}{2} \frac{\nabla \times \mathbf{W}}{c^{2}}+\left(\gamma+\frac{1}{2}\right) \frac{\mathbf{v} \times \nabla U}{c^{2}}
$$

Here, $\mathbf{v}$ is the global coordinate velocity of the rotating body (gyroscope), $\mathbf{Q}$ are the spatial components of the body's four-acceleration and $\mathbf{W}$ the three space-time components of the metric tensor in the comoving system. The three terms on the right hand side describe the well known Thomas precession (first term), Schiff or Lense-Thirring precession (second term) and the geodetic or de Sitter-Fokker precession (third term). The extension-curvature coupling in the post-Newtonian framework of GRT have been discussed in detail in 28]. For recent articles on this coupling in GRT see e.g., Apostolatos 62,63.

It is the purpose of this article to extend and improve the classical PPN framework with parameters $\beta$ and $\gamma$ so to cover also the principal results from the Brumberg-Kopeikin and DSX formalisms. 
Both Brumberg-Kopeikin and DSX approaches use the field equations of GRT in order to construct the local reference system. However, in the PPN formalism we do not have any kind of "parametrized post-Newtonian field equations", but only the metric tensor in the global reference system. To construct a physically adequate local reference system means to find a suitable coordinate transformation which maps the global barycentric metric tensor into a "good" local one having some desired properties. These desired properties in General Relativity read

A. The gravitational field of external bodies is represented only in the form of a relativistic tidal potential which is at least of second order in the local spatial coordinates and coincides with the usual Newtonian tidal potential in the Newtonian limit;

B. The internal gravitational field of the subsystem coincides with the gravitational field of a corresponding isolated source provided that the tidal influence of the external matter is neglected.

These two requirements can simultaneously be satisfied in General Relativity as has been shown in the framework of the Brumberg-Kopeikin and DSX formalisms. It is clear that this fact is closely related with the validity of the Strong Equivalence Principle in GRT. In the PPN formalism due to a possible violation of the Strong Equivalence Principle one expects that either property $\mathbf{A}$ or $\mathbf{B}$ can be satisfied, but not both of them simultaneously.

Both Brumberg-Kopeikin and DSX formalisms rely essentially upon the field equations of general relativity. In contrast to this here we do not want to confine ourselves to a particular theory of gravity and use instead the phenomenological framework of PPN formalism. It is clear that considering a particular theory of gravity might provide a deeper insight into the physical origin of various effects in that particular theory. However, this physical meaning would be valid only in that theory of gravity and cannot be "generalized" onto other theories of gravity even if they give formally the same post-Newtonian metric.

The global barycentric PPN metric is assumed to be a solution of the field equations of any metric gravity theory covered by the PPN formalism. The construction of a physically 
adequate ("good") local reference system involves a suitable coordinate transformation which converts the global barycentric coordinates into "good" local ones. It is clear that if the original barycentric metric represents a solution of the field equations, then the resulting geocentric metric is also a solution of the same equations. The metric tensor of the local reference system is sufficient to discuss both translational and rotational equations of motion in that local reference system since they follow either from the geodetic equations (for test bodies and photons) or from the local equations of motion (for extended bodies). Both kinds of equations require only the metric tensor for their detailed evaluation. No other possible scalar, vector, etc. fields appearing in a particular non-Einsteinian theory of gravity play a role in discussing equations of motion in the PPN formalism. This allows us to construct the local reference system from some geometrical considerations. An attempt to achieve this has been already undertaken by Shahid-Saless and Ashby [64], who introduced a kind of Fermi normal coordinates in a simplified model case of two spherically symmetric nonrotating gravitating bodies. However it is clear that Fermi coordinates in the PPN framework lead to the same principal difficulties when considering the local subsystem with a complicated multipole structure, to which they lead in GRT (see, e.g., 26,22]). We use the much more powerful and elegant approach of the Brumberg-Kopeikin and DSX formalisms.

A practical application of the theory of relativistic astronomical reference systems lies in the construction physically adequate models for modern high-precision astronomical observations (depending on the kind of observations the models involve the coordinate transformations between the reference systems, equations of light propagation in both reference systems as well as equations of translational and rotational motion of extended bodies). However, on the other hand, these observations yield a significant amount of experimental data that can be used for testing metric theories of gravity such as General Relativity. The Parametrized Post-Newtonian (PPN) formalism [1] presently can be considered to represent the standard framework for such tests in the first post-Newtonian approximation. Therefore, it is quite important to have a consistent theory of relativistic astronomical reference systems in the PPN formalism. Besides a purely theoretical interest to construct a "good" proper reference 
system for a massive subsystem (a massive body) in the framework of the PPN formalism, a PPN theory of astronomical reference systems is also important from a rather practical point of view, since presently there is a contradiction between the recommendations of the International Astronomical Union [6] concerning the relativistic reference system to be used for data reduction which are valid only in GRT and the common practice to estimate the PPN parameters (at least $\gamma$ and $\beta$ ) from observations. Moreover, the International Earth Rotation Service being an official service of the IAU recommends in its IERS Conventions [6] to estimate the PPN parameters $\gamma$ and $\beta$ in routine data processing.

This paper focuses mainly on problems of reference systems and celestial mechanics. More specifically, we present

- a new PPN theory of astronomical reference systems (Sections III- \);

- a new description of the local gravitational environment of a body with new PPN massand spin-multipole moments generalizing the Blanchet-Damour moments from [22] (Section VI) and of the tidal forces (Section VII);

- new PPN satellite equations of motion (Section VIII).

- new translational and rotational global equations of motion of $N$ extended, rotating and gravitationally interacting bodies of arbitrary shape and composition with all multipole moments of gravitating bodies with parameters $\beta$ and $\gamma$ (Section $\llbracket$ [X]);

A overall outlook and a summary of our main results are given in Section X. As expected all results that have been listed under 1-4 above are recovered, but now with the help of a new and improved PPN-framework. Some partial results have already been published elsewhere 65 67.

\section{NOTATIONS}

Greek indices $\alpha, \beta, \ldots, \mu, \nu, \ldots$ running from 0 to 3 indicate all four space-time components of the corresponding variable. Latin indices $a, b, \ldots, i, j, \ldots$ run from 1 to 3 and 
refer to three spatial components of the corresponding variable. We use Einstein's summation convention for both types of indices independent of the position of repeated indices: e.g., $x^{i} x^{i} \equiv\left(x^{1}\right)^{2}+\left(x^{2}\right)^{2}+\left(x^{3}\right)^{2}$. We use several special objects: $\delta^{i j}=\operatorname{diag}(1,1,1)$ is the Kronecker delta; $\varepsilon_{i j k}$ is the fully antisymmetic Levi-Civita symbol $\left(\varepsilon_{123}=+1\right)$; $\eta_{\mu \nu}=\operatorname{diag}(-1,+1,+1,+1)$ is the Minkowki flat space metric (we use the signature $(-+++)$ throughout this paper). Capital latin indices $A, B, C, \ldots=1 \ldots N$ (as subscripts or superscripts) refer to the bodies of our $N$-body system: e.g., $\Phi_{E}$ is the value of some variable $\Phi$ related to body $E$.

The global coordinates are designated by $x^{\mu}=\left(c t, x^{i}\right), t$ being coordinate time and $x^{i}$ being spatial coordinates of the global reference system. The global metric is $g_{\mu \nu}\left(x^{\lambda}\right)$ and we systematically use lower case latin letters for the quantities belonging to the global reference system. As in the DSX papers [22,27,28, 33] the space-time indices referring to global coordinates are taken from the second part of the greek alphabet $\mu, \nu, \ldots$ and space indices are taken from the second part of the latin alphabet $i, j, \ldots$. The local coordinates for body $E$ are designated by $X^{\alpha}=\left(c T, X^{a}\right), T$ being coordinate time and $X^{a}$ being spatial coordinates of the global reference system. The local metric is $G_{\alpha \beta}\left(X^{\gamma}\right)$ (do not confuse an index $\beta$ with the PPN parameter $\beta$ ). The space-time indices referring to global coordinates are taken from the first part of the greek alphabet $\alpha, \beta, \ldots$ and space indices are taken from the first part of the latin alphabet $a, b, \ldots$

A comma before an index designates the partial derivative with respect to the corresponding coordinates: $A_{, \mu}=\partial A(t, \mathbf{x}) / \partial x^{\mu}, A_{, i}=\partial A(t, \mathbf{x}) / \partial x^{i}, A_{, \alpha}=\partial A(T, \mathbf{X}) / \partial X^{\alpha}$, $A_{, a}=\partial A(T, \mathbf{X}) / \partial X^{a}$. For partial derivatives with respect to the coordinate times $t$ and $T$ we use $A_{, t}=\partial A(t, \mathbf{x}) / \partial t, A_{, T}=\partial A(T, \mathbf{X}) / \partial T$. A semicolon before a space-time index denotes the corresponding covariant derivative. Parentheses surrounding a group of indices denote symmetrization, e.g., $A_{(i j)}=\frac{1}{2}\left(A_{i j}+A_{j i}\right)$. Brackets surrounding two indices denote antisymmetrization, e.g., $A_{i[j k]}=\frac{1}{2}\left(A_{i j k}-A_{i k j}\right)$. Angle brackets surrounding a group of indices or, alternatively, a caret on top of a tensor symbol denote the symmetric trace-free 
(STF) part of the corresponding object, e.g., $\hat{A}_{a b} \equiv A_{\langle a b\rangle} \equiv S T F_{a b} A_{a b}=A_{(a b)}-\frac{1}{3} \delta^{a b} A_{c c}$. We shall freely lower and raise spatial indices, e.g. $A_{a} \equiv A^{a}$. For sequences of spatial indices we shall use multiindices in the same way as it is done, e.g., in [22]: a spatial multi-index containing $l$ indices is denoted by $L$ ( $K$ for $k$ indices, etc.): $L=i_{1} \ldots i_{l}$ if $L$ refers to global coordinates and $L=a_{1} \ldots a_{l}$ in the case of local ones. We use also $L-1=i_{1} \ldots i_{l-1}$, etc. A multisummation is understood for repeated multiindices: $A_{L} B_{L} \equiv \sum_{i_{1} \ldots i_{l}} A_{i_{1} \ldots i_{l}} B_{i_{1} \ldots i_{l}}$. For a spatial vector $v^{i}$ we denote $v^{L} \equiv v^{i_{1}} v^{i_{2}} \ldots v^{i_{l}}$. For an $L$-order partial derivative we denote $\partial_{L} \equiv \partial_{i_{1}} \ldots \partial_{i_{l}}$.

\section{METRIC TENSORS AND COORDINATE TRANSFORMATIONS}

\section{A. The PPN metric tensor in the global reference system}

We consider a gravitational $N$ body system in an asymptotically flat space-time that can be covered by one single (global) coordinate system $x^{\mu}=\left(c t, x^{i}\right)$ with

$$
\lim _{\substack{|\times| \rightarrow \infty \\ t=\text { const. }}} g_{\mu \nu}=\eta_{\mu \nu}
$$

where $g_{\mu \nu}$ is the metric tensor in the global coordinate system and $\eta_{\mu \nu}=$ $\operatorname{diag}(-1,+1,+1,+1)$ is the flat metric tensor of Minkowski space-time. The metric tensor $g_{\mu \nu}$ in the global PPN reference system is written as

$$
\begin{aligned}
& g_{00}=-1+\frac{2}{c^{2}} w(t, \mathbf{x})-\frac{2}{c^{4}} \beta w^{2}(t, \mathbf{x})+\mathcal{O}\left(c^{-5}\right), \\
& g_{0 i}=-\frac{2(1+\gamma)}{c^{3}} w^{i}(t, \mathbf{x})+\mathcal{O}\left(c^{-5}\right), \\
& g_{i j}=\delta_{i j}\left(1+\frac{2}{c^{2}} \gamma w(t, \mathbf{x})\right)+\mathcal{O}\left(c^{-4}\right) .
\end{aligned}
$$

In accordance with the classical PPN framework we will assume the metric potentials $w$ and $w^{i}$ to obey the equations

$$
\begin{aligned}
w_{, i i}-\frac{1}{c^{2}} w_{, t t} & =-4 \pi G \sigma+\mathcal{O}\left(c^{-4}\right), \\
w_{, j j}^{i} & =-4 \pi G \sigma^{i}+\mathcal{O}\left(c^{-2}\right),
\end{aligned}
$$


where

$$
\begin{aligned}
\sigma & =\frac{1}{c^{2}}\left(T^{00}+\gamma T^{k k}+\frac{1}{c^{2}} T^{00}(3 \gamma-2 \beta-1) w\right)+\mathcal{O}\left(c^{-4}\right), \\
\sigma^{i} & =\frac{1}{c} T^{0 i}+\mathcal{O}\left(c^{-2}\right) .
\end{aligned}
$$

Here, $T^{\mu \nu}$ are the components of energy-momentum tensor in the global reference system and $w$ in (3.5) is needed only to Newtonian order where it coincides with the Newtonian potential. Because of requirement (3.1) $\left(w^{\mu} \equiv\left(w, w^{i}\right)\right)$ we have

$$
\lim _{\substack{|\mathbf{x}| \rightarrow \infty \\ t=\text { const. }}} w^{\mu}(t, \mathbf{x})=0
$$

and the solution of $(3.3)-(3.4)$ can be written in the form

$$
w^{\mu}(t, \mathbf{x})=G \int \frac{\sigma^{\mu}\left(t, \mathbf{x}^{\prime}\right)}{\left|\mathbf{x}-\mathbf{x}^{\prime}\right|} d^{3} x^{\prime}+\frac{1}{2 c^{2}} G \frac{\partial^{2}}{\partial t^{2}} \int \sigma^{\mu}\left(t, \mathbf{x}^{\prime}\right)\left|\mathbf{x}-\mathbf{x}^{\prime}\right| d^{3} x^{\prime}+\mathcal{O}\left(c^{-4}\right) \text {. }
$$

The expressions above are valid independently of the particular form of the energymomentum tensor. This is no more than a formal way to specify the global PPN metric tensor. If matter is described as an ideal fluid or stressed continuum the metric (3.2)-(3.8) coincides with the version of the PPN formalism described in [1] as well as with the version discussed in [38], provided that only two parameters $\gamma$ and $\beta$ are retained in both versions. Effectively we consider only those theories of gravity which produce the metric (3.2)-(3.6) in the first post-Newtonian approximation for some form of the energy-momentum tensor.

Eqs. (3.3)-(3.6) can be considered as "effective" or "phenomenological" post-Newtonian field equations in the global reference system. These equations reduce to the Einstein field equations for $\gamma=\beta=1$. It is, however, clear that these phenomenological field equations have nothing to do with the actual field equations of a specific theory of gravity covered by the PPN formalism.

The system under study is supposed to be spatially bounded and isolated which means that $T^{\mu \nu}=0$ sufficiently far from the origin. We suppose also that the matter in concentrated in spatially separated blobs (bodies) and $T^{\mu \nu}$ vanishes outside these blobs. One of such blobs is selected to define a local subsystem and the matter inside the blob is referred below to 
as internal matter. All other matter is called external. Any subsystem is characterized by a world tube $V$ which encompasses the internal matter. Since the subsystems are supposed to be spatially separated we choose the world tube $V$ in such a way that $T^{\mu \nu}=0$ on the boundary of $V$. In the following we will concentrate upon one of these bodies that we will call "Earth" (indicated by subscript $E$ ).

The potentials $w^{\mu}$ are defined by (3.8) as volume integrals over the whole 3 -space. Therefore, splitting the area of integration into the volume $V$ of the body, for which we want to construct a local reference system, and the remaining part of space, we split $w^{\mu}$ into internal potentials (potentials of the body under consideration) and external ones (potentials due to the other bodies):

$$
\begin{aligned}
w(t, \mathbf{x}) & =w_{\mathrm{E}}(t, \mathbf{x})+\bar{w}(t, \mathbf{x}), \\
w^{i}(t, \mathbf{x}) & =w_{\mathrm{E}}^{i}(t, \mathbf{x})+\bar{w}^{i}(t, \mathbf{x}) .
\end{aligned}
$$

The internal potentials $w_{\mathrm{E}}^{\mu}=\left(w_{\mathrm{E}}, w_{\mathrm{E}}^{i}\right)$ are defined as

$$
w_{\mathrm{E}}^{\mu}(t, \mathbf{x})=G \int_{V} \frac{\sigma^{\mu}\left(t, \mathbf{x}^{\prime}\right)}{\left|\mathbf{x}-\mathbf{x}^{\prime}\right|} d^{3} x^{\prime}+\frac{1}{2 c^{2}} G \frac{\partial^{2}}{\partial t^{2}} \int_{V} \sigma^{\mu}\left(t, \mathbf{x}^{\prime}\right)\left|\mathbf{x}-\mathbf{x}^{\prime}\right| d^{3} x^{\prime} .
$$

We adopt a harmonic-like gauge for the global PPN metric tensor. Precisely speaking, the global metric tensor satisfies the usual harmonic gauge $\left(g=\operatorname{det}\left(g_{\mu \nu}\right)\right)$

$$
\frac{\partial}{\partial x^{\alpha}}\left((-g)^{1 / 2} g^{\alpha \beta}\right)=0
$$

in case of General Relativity $\beta=\gamma=1$. This requires

$$
w_{, t}+w_{, i}^{i}=\mathcal{O}\left(c^{-2}\right)
$$

which is also compatible with the definitions of $w$ and $w^{i}$, and the Newtonian continuity equation

$$
\sigma_{, t}+\sigma_{, i}^{i}=\mathcal{O}\left(c^{-2}\right)
$$

Since the energy-momentum tensor $T^{\mu \nu}$ is supposed to vanish on the boundary of the world tube $V$ encompassing the subsystem, Eq. (3.13) allows one to formulate (3.12) separately for internal and external potentials 


$$
\begin{gathered}
w_{E, t}+w_{E, i}^{i}=\mathcal{O}\left(c^{-2}\right), \\
\bar{w}_{, t}+\bar{w}_{, i}^{i}=\mathcal{O}\left(c^{-2}\right) .
\end{gathered}
$$

The metric (3.2)-(3.8) is equivalent to the PPN metric in [1] up to a trivial gauge transformation. The transformations between our reference system $\left(t, x^{i}\right)$ and the PPN reference system in the standard post-Newtonian gauge $\left(t_{\mathrm{PN}}, x_{\mathrm{PN}}^{i}\right)$ reads

$$
\begin{aligned}
t_{\mathrm{PN}} & =t-\frac{1}{c^{4}} \chi_{, t}+\mathcal{O}\left(c^{-5}\right), \\
x_{\mathrm{PN}}^{i} & =x^{i},
\end{aligned}
$$

where $\chi$ is the superpotential, here defined by

$$
\chi=\frac{1}{2} G \int \sigma\left(t, \mathbf{x}^{\prime}\right)\left|\mathbf{x}-\mathbf{x}^{\prime}\right| d^{3} x^{\prime}+\mathcal{O}\left(c^{-2}\right),
$$

so that

$$
\chi_{, i i}=w+\mathcal{O}\left(c^{-2}\right)
$$

The transformation of the results derived below into the standard PPN gauge represents no principal difficulty and can be done in exactly the same way as it has been done in Section $\mathrm{V}$ of [29].

\section{B. Energy-momentum tensor for an ideal fluid}

Although, as noted above, our formalism is not restricted to any particular form of the energy-momentum tensor, the standard PPN formalism (see, e.g., [1]) is elaborated for the case of an ideal fluid and it is interesting to specify our formalism for this simplified case. The energy-momentum tensor of an ideal fluid in the global asymptotically flat reference system $\left(t, x^{i}\right)$ reads

$$
\begin{aligned}
T^{00} & =\rho c^{2}\left[1+\frac{1}{c^{2}}\left(v^{2}+\Pi+2 U\right)\right]+\mathcal{O}\left(c^{-2}\right) \\
T^{0 i} & =\rho c v^{i}\left[1+\frac{1}{c^{2}}\left(v^{2}+\Pi+2 U\right)\right]+\frac{1}{c} p v^{i}+\mathcal{O}\left(c^{-3}\right), \\
T^{i j} & =\rho v^{i} v^{j}+\delta^{i j} p+\mathcal{O}\left(c^{-2}\right)
\end{aligned}
$$


Here $v^{i}=v^{i}(t, \mathbf{x})$ is the velocity of matter, $\Pi(t, \mathbf{x})$ is the specific energy density (ratio of energy density to rest-mass density), $p(t, \mathbf{x})$ is the isotropic pressure, $\rho(t, \mathbf{x})$ is the invariant density (that is, the density invariant under coordinate transformations from $(t, \mathbf{x})$ to any $(\tilde{t}, \tilde{\mathbf{x}}): \rho(t, \mathbf{x})=\rho(\tilde{t}, \tilde{\mathbf{x}}))$. In many cases it is more convenient to express the energymomentum tensor in terms of $\rho^{*}(t, \mathbf{x})$,

$$
\rho^{*}(t, \mathbf{x})=\rho(t, \mathbf{x})(-g)^{1 / 2} \frac{c d t}{d s}=\rho\left[1+\frac{1}{c^{2}}\left(\frac{1}{2} v^{2}+3 \gamma U\right)+\mathcal{O}\left(c^{-4}\right)\right] .
$$

For the density $\rho^{*}$ a Newtonian-like continuity equation holds to post-Newtonian accuracy

$$
\frac{\partial}{\partial t} \rho^{*}+\frac{\partial}{\partial x^{i}}\left(\rho^{*} v^{i}\right)=\mathcal{O}\left(c^{-4}\right) .
$$

In terms of $\rho^{*}$ the energy-momentum tensor reads

$$
\begin{aligned}
T^{00} & =\rho^{*} c^{2}\left[1+\frac{1}{c^{2}}\left(\frac{1}{2} v^{2}+\Pi-(3 \gamma-2) U\right)\right]+\mathcal{O}\left(c^{-2}\right), \\
T^{0 i} & =\rho^{*} c v^{i}\left[1+\frac{1}{c^{2}}\left(\frac{1}{2} v^{2}+\Pi-(3 \gamma-2) U\right)\right]+\frac{1}{c} p v^{i}+\mathcal{O}\left(c^{-3}\right), \\
T^{i j} & =\rho^{*} v^{i} v^{j}+\delta^{i j} p+\mathcal{O}\left(c^{-2}\right) .
\end{aligned}
$$

Here and above $U$ is the Newtonian-like gravitational potential

$$
U(t, \mathbf{x})=G \int \frac{\rho^{*}\left(t, \mathbf{x}^{\prime}\right)}{\left|\mathbf{x}-\mathbf{x}^{\prime}\right|} d^{3} x^{\prime} .
$$

For an ideal fluid the potentials $w$ and $w^{i}$ can then be expressed as

$$
\begin{aligned}
w & =U+\frac{1}{c^{2}}\left(\left(\gamma+\frac{1}{2}\right) \Phi_{1}+(1-2 \beta) \Phi_{2}+\Phi_{3}+3 \gamma \Phi_{4}+\chi_{, t t}\right), \\
w^{i} & =G \int \frac{\rho^{*}\left(t, \mathbf{x}^{\prime}\right) v^{i}\left(t, \mathbf{x}^{\prime}\right)}{\left|\mathbf{x}-\mathbf{x}^{\prime}\right|} d^{3} x^{\prime}+\mathcal{O}\left(c^{-2}\right), \\
\Phi_{1} & =G \int \frac{\rho^{*}\left(t, \mathbf{x}^{\prime}\right) \dot{x}^{\prime 2}}{\left|\mathbf{x}-\mathbf{x}^{\prime}\right|} d^{3} x^{\prime}, \\
\Phi_{2} & =G \int \frac{\rho^{*}\left(t, \mathbf{x}^{\prime}\right) U\left(t, \mathbf{x}^{\prime}\right)}{\left|\mathbf{x}-\mathbf{x}^{\prime}\right|} d^{3} x^{\prime}, \\
\Phi_{3} & =G \int \frac{\rho^{*}\left(t, \mathbf{x}^{\prime}\right) \Pi\left(t, \mathbf{x}^{\prime}\right)}{\left|\mathbf{x}-\mathbf{x}^{\prime}\right|} d^{3} x^{\prime}, \\
\Phi_{4} & =G \int \frac{p\left(t, \mathbf{x}^{\prime}\right)}{\left|\mathbf{x}-\mathbf{x}^{\prime}\right|} d^{3} x^{\prime} .
\end{aligned}
$$


Since all the potentials $(3.23),(3.26)-(3.29)$ and (3.17) are again some volume integrals, we can split them into local parts where the integration is performed over a world tube $V$ covering the subsystem for which the local RS is being constructed and external ones (the integration is performed only outside of $V$ )

$$
\begin{aligned}
U & =U_{\mathrm{E}}+\bar{U}, \\
\Phi_{i} & =\Phi_{i}^{\mathrm{E}}+\bar{\Phi}_{i}, i=1,2,3,4, \\
\chi & =\chi^{\mathrm{E}}+\bar{\chi} .
\end{aligned}
$$

For example, the definition of $U_{E}$ reads

$$
U_{\mathrm{E}}(t, \mathbf{x})=G \int_{V} \frac{\rho^{*}\left(t, \mathbf{x}^{\prime}\right)}{\left|\mathbf{x}-\mathbf{x}^{\prime}\right|} d^{3} x^{\prime}
$$

\section{The PPN metric tensor in the local geocentric system}

We assume that the metric tensor $G_{\mu \nu}(T, \mathbf{X})$ in the local reference system $X^{\mu}=\left(c T, X^{a}\right)$ has the same functional form as the metric in the global reference system

$$
\begin{aligned}
G_{00} & =-1+\frac{2}{c^{2}} W(T, \mathbf{X})-\frac{2}{c^{4}} \beta W^{2}(T, \mathbf{X})+\mathcal{O}\left(c^{-5}\right) \\
G_{0 a} & =-\frac{2(1+\gamma)}{c^{3}} W^{a}(T, \mathbf{X})+\mathcal{O}\left(c^{-5}\right) \\
G_{a b} & =\delta_{a b}\left(1+\frac{2}{c^{2}} \gamma W(T, \mathbf{X})\right)+\mathcal{O}\left(c^{-4}\right)
\end{aligned}
$$

and the potentials $W$ and $W^{i}$ entering into (3.32) admit a representation in the form

$$
\begin{aligned}
W(T, \mathbf{X}) & =W_{\mathrm{E}}(T, \mathbf{X})+Q_{a}(T) X^{a}+W_{\mathrm{T}}(T, \mathbf{X})+\frac{1}{c^{2}} \Psi(T, \mathbf{X}), \\
W^{a}(T, \mathbf{X}) & =W_{\mathrm{E}}^{a}(T, \mathbf{X})+\frac{1}{2} \varepsilon_{a b c} C_{b}(T) X^{c}+W_{\mathrm{T}}^{a}(T, \mathbf{X}) .
\end{aligned}
$$

Here $W_{T}$ and $W_{T}^{a}$ are external potentials representing tidal fields of the other bodies of the system and are assumed to be $\mathcal{O}\left(\mathbf{X}^{2}\right)$. The two arbitrary functions $Q_{a}$ and $C_{a}$ have a clear physical meaning which will be discussed below. The local internal gravitational potentials are represented by three other functions $W_{\mathrm{E}}, W_{\mathrm{E}}^{a}$ and $\Psi$. The potentials $W_{\mathrm{E}}$ and $W_{\mathrm{E}}^{a}$ are 
supposed to have the same functional form as their counterparts $w_{\mathrm{E}}$ and $w_{\mathrm{E}}^{i}$, but expressed in local coordinates:

$$
\begin{aligned}
& W_{\mathrm{E}}= G \int_{V} \Sigma\left(T, \mathbf{X}^{\prime}\right) \frac{1}{\left|\mathbf{X}-\mathbf{X}^{\prime}\right|} d^{3} X^{\prime} \\
&+\frac{1}{2 c^{2}} G \frac{\partial^{2}}{\partial T^{2}} \int_{V} \Sigma\left(T, \mathbf{X}^{\prime}\right)\left|\mathbf{X}-\mathbf{X}^{\prime}\right| d^{3} X^{\prime}+\mathcal{O}\left(c^{-4}\right), \\
& W_{\mathrm{E}}^{a}= G \int_{V} \Sigma^{a}\left(T, \mathbf{X}^{\prime}\right) \frac{1}{\left|\mathbf{X}-\mathbf{X}^{\prime}\right|} d^{3} X^{\prime}+\mathcal{O}\left(c^{-2}\right), \\
& \Sigma= \frac{1}{c^{2}}\left(\mathcal{T}^{00}+\gamma \mathcal{T}^{a a}+\frac{1}{c^{2}} \mathcal{T}^{00}(3 \gamma-2 \beta-1) W\right)+\mathcal{O}\left(c^{-4}\right), \\
& \Sigma^{a}=\frac{1}{c} \mathcal{T}^{0 a}+\mathcal{O}\left(c^{-2}\right),
\end{aligned}
$$

where $\mathcal{T}^{\mu \nu}$ is the energy-momentum tensor in the local reference system $\left(T, X^{a}\right)$. Finally, the function $\Psi(T, \mathbf{X})$ represents any possible deviation of the actual internal gravitational field as seen in the local reference system from the form (3.35)-(3.36). Clearly the appearance of $\Psi$ is related with a violation of the Strong Equivalence Principle which makes it impossible

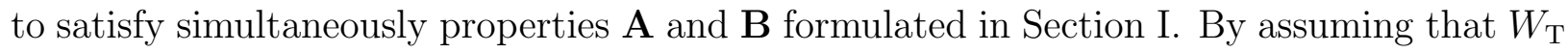
and $W_{\mathrm{T}}^{a}$ are $\sim \mathcal{O}\left(\mathbf{X}^{2}\right)$ we assume that property $\mathbf{A}$ is satisfied. For that reason property $\mathbf{B}$ will be violated resulting in the appearance of the additional quantity $\Psi$.

The local metric is also supposed to satisfy the harmonic gauge $\left(\mathcal{G}=\operatorname{det}\left(G_{\alpha \beta}\right)\right)$

$$
\frac{\partial}{\partial X^{\alpha}}\left((-\mathcal{G})^{1 / 2} G^{\alpha \beta}\right)=0
$$

in case of General Relativity $(\beta=\gamma=1)$. This requires

$$
W_{, T}+W_{, a}^{a}=\mathcal{O}\left(c^{-2}\right)
$$

From (3.35) and (3.36), the Newtonian continuity equation

$$
\Sigma_{, T}+\Sigma_{, a}^{a}=\mathcal{O}\left(c^{-2}\right)
$$

and from the assumption that the energy-momentum tensor (and, therefore, $\Sigma$ and $\Sigma^{a}$ ) vanishes on the boundary of the world tube $V$ it follows 


$$
W_{\mathrm{E}, T}+W_{\mathrm{E}, a}^{a}=\mathcal{O}\left(c^{-2}\right) .
$$

From $(3.40)$ and $(3.42)$ one finds

$$
\dot{Q}_{a} X^{a}+W_{\mathrm{T}, T}+W_{\mathrm{T}, a}^{a}=\mathcal{O}\left(c^{-2}\right) .
$$

Let us note that at this point there is no way to prove that a local reference system with all these properties exists. Unlike the case of General Relativity we do not have field equations that would allow us to prove some properties of the local reference system. We just formulate the desired properties of the local reference system and then prove that it is indeed possible to construct such a system.

\section{Coordinate transformations between the global and local reference systems}

The results of the Brumberg-Kopeikin and DSX formalisms (see, e.g., Theorems 1 and 2 of [22]) allow us to write the transformations between the global and local reference systems in the form

$$
\begin{aligned}
& T=t-\frac{1}{c^{2}}\left(A+v_{\mathrm{E}}^{i} r_{\mathrm{E}}^{i}\right)+\frac{1}{c^{4}}\left(B+B^{i} r_{\mathrm{E}}^{i}+B^{i j} r_{\mathrm{E}}^{i} r_{\mathrm{E}}^{j}+C(t, \mathbf{x})\right)+\mathcal{O}\left(c^{-5}\right), \\
& X^{a}=R_{j}^{a}\left(r_{\mathrm{E}}^{j}+\frac{1}{c^{2}}\left(\frac{1}{2} v_{\mathrm{E}}^{j} v_{\mathrm{E}}^{k} r_{\mathrm{E}}^{k}+D^{j k} r_{\mathrm{E}}^{k}+D^{j k l} r_{\mathrm{E}}^{k} r_{\mathrm{E}}^{l}\right)\right)+\mathcal{O}\left(c^{-4}\right),
\end{aligned}
$$

where $r_{\mathrm{E}}^{i}=x^{i}-x_{\mathrm{E}}^{i}(t), x_{\mathrm{E}}^{i}(t)$ is the coordinates of the origin of the local reference system relative to the global one, and $v_{\mathrm{E}}^{i}=d x_{\mathrm{E}}^{i} / d t$ and $a_{\mathrm{E}}^{i}=d^{2} x_{\mathrm{E}}^{i} / d t^{2}$ are its velocity and acceleration, respectively. The functions $A(t), B(t), B^{i}(t), B^{i j}(t), D^{i j}(t), D^{i j k}(t), R_{j}^{a}(t)$ (being

a rotational (orthogonal) matrix) and $C(t, \mathbf{x}) \sim \mathcal{O}\left(r_{\mathrm{E}}^{3}\right)$ are some unknown functions to be determined with the aid of matching of the two metric tensors.

The fact that the metric (3.2) can be transformed into the metric (3.32) with the help of the transformations (3.44)-(3.45) is not obvious from the beginning and has to be verified.

\section{MATCHING OF THE GLOBAL AND LOCAL PPN METRIC TENSORS}

Matching of the metric tensors (3.2) and (3.32) 


$$
g_{\varepsilon \lambda}(t, \mathbf{x})=\frac{\partial X^{\mu}}{\partial x^{\varepsilon}} \frac{\partial X^{\nu}}{\partial x^{\lambda}} G_{\mu \nu}(T, \mathbf{X})
$$

where the Jacobian $\partial X^{\mu} / \partial x^{\varepsilon}$ has to be determined from the coordinate transformation (3.44)-(3.45), yields the explicit expressions for the unknown functions in the coordinate transformations as well as in the local metric. The matching is performed in four steps, considering
A) terms of order $\mathcal{O}\left(c^{-2}\right)$ in $g_{00}(\varepsilon=\lambda=0$ in (4.1));
B) terms of order $\mathcal{O}\left(c^{-2}\right)$ in $g_{i j}(\varepsilon=i, \lambda=j$ in $(4.1))$;
C) terms of order $\mathcal{O}\left(c^{-3}\right)$ in $g_{0 i}(\varepsilon=i, \lambda=0$ in $(4.1))$;
D) terms of order $\mathcal{O}\left(c^{-4}\right)$ in $g_{00}(\varepsilon=\lambda=0$ in (4.1)).

At each step of the matching procedure we equate separately four kind of terms in the leftand right-hand sides of (4.1):

1) terms containing the internal potentials of either global or local metric;

2) external terms independent of local spatial coordinates (that is, the terms which are functions of time only);

3) external terms linear with respect to local coordinates $\mathbf{X}$;

4) external terms of at least second order with respect to local coordinates $\mathbf{X}$.

This procedure allows us to derive the following results.

\section{A. Matching of order $\mathcal{O}\left(c^{-2}\right)$ in $g_{00}$}

1) Internal terms give the Newtonian transformation between the internal potentials in the global and local reference systems

$$
W_{\mathrm{E}}(T, \mathbf{X})=w_{\mathrm{E}}(t, \mathbf{x})+\mathcal{O}\left(c^{-2}\right)
$$


It is easy to see that (4.2) is in agreement with the definitions of $w_{E}(t, \mathbf{x})$ and $W_{E}(u, \mathbf{w})$ (see, (3.10) and (3.35)).

2) External terms of order $|\mathbf{X}|^{0}$ give

$$
\frac{d}{d t} A=\frac{1}{2} v_{E}^{2}+\bar{w}\left(\mathbf{x}_{E}\right)
$$

Here and below for any function $\Phi(t, \mathbf{x})$ we use the shorthand notation $\Phi\left(\mathbf{x}_{E}\right) \equiv \Phi\left(t, \mathbf{x}_{E}(t)\right)$.

3) External terms of order $|\mathbf{X}|^{1}$ lead to the Newtonian equations of motion of the center of mass of the local system relative to the global reference system

$$
a_{E}^{i}(t)=\bar{w}_{, i}\left(\mathbf{x}_{E}\right)-R_{i}^{a} Q_{a}+\mathcal{O}\left(c^{-2}\right)
$$

4) External terms of order $|\mathbf{X}|^{l}, l \geq 2$ yield the expression for the external tidal potential in the local metric

$$
W_{\mathrm{T}}(T, \mathbf{X})=\bar{w}(t, \mathbf{x})-\bar{w}\left(\mathbf{x}_{E}\right)-\bar{w}_{, i}\left(\mathbf{x}_{E}\right) r_{E}^{i}+\mathcal{O}\left(c^{-2}\right)
$$

Here and below for any function $\Phi(t, \mathbf{x})$ the shorthand notation $\Phi_{, i}\left(\mathbf{x}_{E}\right)$ denotes $\partial \Phi(t, \mathbf{x}) / \partial x^{i}$ evaluated at $\mathbf{x}=\mathbf{x}_{E}(t)$.

\section{B. Matching of order $\mathcal{O}\left(c^{-2}\right)$ in $g_{i j}$}

1) Internal terms are matched automatically due to (4.2).

2) External terms of order $|\mathbf{X}|^{0}$ give

$$
D^{i j}(t)=\delta^{i j} \gamma \bar{w}\left(\mathbf{x}_{E}\right)
$$

3) External terms of order $|\mathbf{X}|^{1}$ give

$$
D^{i j k}(t)=\frac{1}{2} \gamma\left(\delta^{i j} a_{E}^{k}+\delta^{i k} a_{E}^{j}-\delta^{j k} a_{E}^{i}\right)
$$


Let us note here that this expression for $D^{i j k}$ follows from the isotropic form of $g_{i j}$ and $G_{a b}$ and its relation to $g_{00}$ and $G_{00}$, respectively (this was proven by Theorem 2 of [22] in the case $\gamma=1)$.

4) External terms of order $|\mathbf{X}|^{l}, l \geq 2$ are matched automatically due to (4.5).

\section{Matching of order $\mathcal{O}\left(c^{-3}\right)$ in $g_{0 i}$}

1) Equating internal terms results in the expression for the local vector potential

$$
W_{E}^{a}(T, \mathbf{X})=R_{i}^{a}\left(w_{E}^{i}(t, \mathbf{x})-v_{E}^{i} w_{E}(t, \mathbf{x})\right)+\mathcal{O}\left(c^{-2}\right)
$$

This agrees with the definitions $(3.10)$ and (3.36) of $w_{E}^{i}(t, \mathbf{x})$ and $W_{E}^{a}(T, \mathbf{X})$ and the relation

$$
\Sigma^{a}=R_{i}^{a}\left(\sigma^{i}-v_{E}^{i} \sigma\right)+\mathcal{O}\left(c^{-2}\right)
$$

which can be derived the definitions (3.38), (3.5)-(3.6) of $\Sigma^{a}, \sigma^{i}$ and $\sigma$ with the help of the coordinate transformations (3.44)-(3.45).

2) External terms of order $|\mathbf{X}|^{0}$ require

$$
B^{i}(t)=-\frac{1}{2} v_{E}^{2} v_{E}^{i}+2(1+\gamma) \bar{w}^{i}\left(\mathbf{x}_{E}\right)-(2 \gamma+1) v_{E}^{i} \bar{w}\left(\mathbf{x}_{E}\right)
$$

3) External terms of order $|\mathbf{X}|^{1}$ lead to

$$
\begin{aligned}
B^{i j}(t)= & \left.-v_{E}^{(i} R_{j)}^{a} Q^{a}+(1+\gamma) \bar{w}^{(i, j)}\left(\mathbf{x}_{E}\right)-\gamma v_{E}^{(i} \bar{w}^{j} j\right)+\frac{1}{2} \gamma \delta^{i j} \dot{\bar{w}}\left(\mathbf{x}_{E}\right), \\
c^{2} R_{i}^{a} \dot{R}_{j}^{a}= & -(1+\gamma) \varepsilon_{i j k} R_{k}^{a} C_{a} \\
& -2(1+\gamma) \bar{w}^{[i, j]}\left(\mathbf{x}_{\mathrm{E}}\right)+(1+2 \gamma) v_{\mathrm{E}}^{[i} \bar{w}_{, j]}\left(\mathbf{x}_{\mathrm{E}}\right)+v_{\mathrm{E}}^{[i} R_{j]}^{a} Q_{a}+\mathcal{O}\left(c^{-2}\right) .
\end{aligned}
$$

Eq. (4.12) relates the two functions $C_{a}$ and $R_{i}^{a}$. These functions together describe the spatial orientation of the local reference system with respect to the global one. According to (4.12) one can choose $C_{a}(T)$ so that $R_{i}^{a}=\delta^{a}{ }_{i}$ and the resulting local reference system does not 
rotate relative to the global one, i.e., the local reference system is kinematically non-rotating. Another possible choice $C_{a}=0$ results in a dynamically nonrotating local reference system, and the orthogonal matrix $R^{a}{ }_{i}$ in the transformations of the spatial coordinates represents the well-known de Sitter, Lense-Thirring and Thomas precessions. Note that (4.12) contains the well-known results for Lense-Thirring and de Sitter precessions in the PPN formalism (the second and third terms on the right-hand side, respectively). On the other hand, the fourth term represents Thomas precession appearing in special relativity and therefore, must be independent of the PPN parameters, which is indeed the case. The first terms in $B^{i}$ and $B^{i j}$ (Eqs. (4.10) and (4.11)) come also from special relativity theory and are also independent of $\beta$ and $\gamma$.

4) External terms of order $|\mathbf{X}|^{l}, l \geq 2$ give the external tidal vector potential in the local reference system

$$
\begin{aligned}
& W_{\mathrm{T}}^{a}(T, \mathbf{X})=R_{i}^{a}\left\{\bar{w}^{i}(t, \mathbf{x})-\bar{w}^{i}\left(\mathbf{x}_{\mathrm{E}}\right)-\bar{w}_{, j}^{i}\left(\mathbf{x}_{\mathrm{E}}\right) r_{\mathrm{E}}^{j}-v_{\mathrm{E}}^{i} W_{\mathrm{T}}(T, \mathbf{X})\right. \\
& \left.+\frac{1}{2(1+\gamma)}\left(\dot{D}^{i j k} r_{E}^{j} r_{E}^{k}-C_{, i}\right)\right\}+\mathcal{O}\left(c^{-2}\right) .
\end{aligned}
$$

Eq. (4.13) together with (3.15) and (3.43) leads to a Poisson-type equation for the unknown function $C(t, \mathbf{x})$

$$
C_{, i i}=2 \dot{D}^{i i k} r_{E}^{k}-2(1+\gamma) \dot{a}_{E}^{k} r_{E}^{k}=(\gamma-2) \dot{a}_{E}^{k} r_{E}^{k}
$$

This equation does not fix $C(t, \mathbf{x})$ uniquely, but only up to a harmonic function. This fact reflects some ambiguity in constructing the local reference system in the form (3.32) $-($ (3.34). Assuming the function $C$ together with its first and second partial derivatives with respect to $X^{a}$ to be equal to zero at $X^{a}=0$, the general solution of (4.14) reads

$$
C=C_{1}+C_{2}
$$

where

$$
C_{1}(t, \mathbf{x})=\frac{\gamma-2}{10} r_{E}^{2}\left(\dot{a}_{E}^{i} r_{E}^{i}\right)
$$


is the particular solution of (4.14), and

$$
C_{2}(t, \mathbf{x})=\sum_{p=3}^{\infty} \frac{1}{p !} V_{a_{1} \ldots a_{p}} r_{E}^{a_{1}} \ldots r_{E}^{a_{p}}
$$

Here $V_{a_{1} \ldots a_{p}}(t), p \geq 3$ is a family of arbitrary STF tensors. Any closed-form solution of (4.14) leads to a closed-form metric tensor of the local reference system. E.g., we might choose the simplest solution $C=C_{1}=(\gamma-2) r_{E}^{2}\left(\dot{a}_{E}^{i} r_{E}^{i}\right) / 10$ corresponding to $V_{a_{1} \ldots a_{p}}(t) \equiv 0$. In this case the tidal vector-potential reads

$$
\begin{aligned}
W_{\mathrm{T}}^{a}(T, \mathbf{X})=R_{i}^{a}\{ & \bar{w}^{i}(t, \mathbf{x})-\bar{w}^{i}\left(\mathbf{x}_{\mathrm{E}}\right)-\bar{w}_{, j}^{i}\left(\mathbf{x}_{\mathrm{E}}\right) r_{\mathrm{E}}^{j}-v_{\mathrm{E}}^{i} W_{\mathrm{T}}(T, \mathbf{X}) \\
& \left.+\frac{2 \gamma+1}{5(1+\gamma)} r_{E}^{i}\left(\dot{a}_{E}^{k} r_{E}^{k}\right)-\frac{3 \gamma-1}{10(1+\gamma)} \dot{a}_{E}^{i} \mathbf{r}_{E}^{2}\right\}+\mathcal{O}\left(c^{-2}\right)
\end{aligned}
$$

Note once again that the factor $\gamma+1$ in denominators of (4.13) and (4.18) cancels with the same one in the numerator of (3.32) and no rational functions of $\gamma$ appear in the metric tensor.

It is easy to see from (4.13)-(4.14) that independent of $C_{2}$ the tidal vector potential $W_{T}^{i}$ satisfies the equation

$$
W_{T, b b}^{a}=\frac{1-\gamma}{1+\gamma} R_{i}^{a} \dot{a}_{E}^{i}+\mathcal{O}\left(c^{-2}\right)
$$

\section{Matching of order $\mathcal{O}\left(c^{-4}\right)$ in $g_{00}$}

1) Matching of both terms $\mathcal{O}\left(c^{-2}\right)$ and $\mathcal{O}\left(c^{-4}\right)$ in $g_{00}$ gives

$$
\begin{aligned}
W_{\mathrm{E}}(T, \mathbf{X})+\frac{1}{c^{2}} \Psi(T, \mathbf{X})= & w_{\mathrm{E}}(t, \mathbf{x})\left(1+\frac{1}{c^{2}}(\gamma+1) v_{E}^{2}\right) \\
& -\frac{2(\gamma+1)}{c^{2}} w_{\mathrm{E}}^{i}(t, \mathbf{x}) v_{E}^{i} \\
& -\frac{2(\beta-1)}{c^{2}} w_{\mathrm{E}}(t, \mathbf{x})\left(\bar{w}\left(\mathbf{x}_{E}\right)+a_{E}^{i} r_{E}^{i}\right)+\mathcal{O}\left(c^{-4}\right),
\end{aligned}
$$

generalizing (4.2) to post-Newtonian accuracy. On the other hand, one can relate the local internal potential $W_{\mathrm{E}}$ with the global ones $w_{\mathrm{E}}$ and $w_{\mathrm{E}}^{i}$ directly by using their definitions 
(3.10) and (3.35). To this end, from the definitions (3.37), (3.5)-(3.6) of $\Sigma, \sigma^{i}$ and $\sigma$, and the coordinate transformations (3.44)-(3.45) one derives

$$
\begin{aligned}
\Sigma(T, \mathbf{X})= & \sigma(t, \mathbf{x})\left(1+\frac{1}{c^{2}}\left((1+\gamma) v_{E}^{2}+(2 \beta-3 \gamma-1)\left(\bar{w}\left(\mathbf{x}_{E}\right)+a_{E}^{k} r_{E}^{k}\right)\right)\right) \\
& -\frac{1}{c^{2}} 2(1+\gamma) v_{E}^{i} \sigma^{i}+\mathcal{O}\left(c^{-4}\right) .
\end{aligned}
$$

Using this the following result generalizing Theorem 5 of [22 is found

$$
\begin{aligned}
W_{\mathrm{E}}(T, \mathbf{X})= & w_{\mathrm{E}}(t, \mathbf{x})\left(1+\frac{1}{c^{2}}(\gamma+1) v_{E}^{2}\right) \\
& -\frac{2(\gamma+1)}{c^{2}} w_{\mathrm{E}}^{i}(t, \mathbf{x}) v_{E}^{i} \\
& +\frac{1}{c^{2}}(2 \beta-\gamma-1) w_{\mathrm{E}}(t, \mathbf{x})\left(\bar{w}\left(\mathbf{x}_{E}\right)+a_{E}^{i} r_{E}^{i}\right) \\
& -\frac{1}{c^{2}}(4 \beta-\gamma-3) \chi_{, i}^{\mathrm{E}} a_{E}^{i}+\mathcal{O}\left(c^{-4}\right) .
\end{aligned}
$$

Here,

$$
\chi^{\mathrm{E}}(t, \mathbf{x})=\frac{1}{2} G \int_{V} \sigma\left(t, \mathbf{x}^{\prime}\right)\left|\mathbf{x}-\mathbf{x}^{\prime}\right| d^{3} x^{\prime}+\mathcal{O}\left(c^{-2}\right)
$$

and

$$
\chi^{\mathrm{E}}(T, \mathbf{X})=\frac{1}{2} G \int_{V} \Sigma\left(T, \mathbf{X}^{\prime}\right)\left|\mathbf{X}-\mathbf{X}^{\prime}\right| d^{3} X^{\prime}+\mathcal{O}\left(c^{-2}\right)=\chi^{\mathrm{E}}(t, \mathbf{x})+\mathcal{O}\left(c^{-2}\right) .
$$

A comparison of $(4.20)$ with $(4.22)$ shows that

$$
\Psi(T, \mathbf{X})=-\eta\left(w_{\mathrm{E}}\left(\bar{w}\left(\mathbf{x}_{E}\right)+a_{E}^{i} r_{E}^{i}\right)-\chi_{, i}^{\mathrm{E}} a_{E}^{i}\right)+\mathcal{O}\left(c^{-2}\right)
$$

where

$$
\eta=4 \beta-\gamma-3
$$

is the well-known Nordtvedt parameter indicating a violation of the Strong Equivalence Principle in the PPN formalism. The fact that $\Psi$ is not equal to zero unless $\eta=0$ means that requirement $\mathbf{B}$ from Introduction is violated and the internal gravitational field in our local reference system does not have the same form as the corresponding solution for an isolated 
body. This has a profound physical meaning (see, also Section $\square$ for further discussion) and is directly related with the violation of the equivalence principle for those theories where $\eta \neq 0$. In case of a system consisting of two spherically symmetrical nonrotating masses the term $\Psi$ is equal exactly to the 6th and 7 th terms in $g_{00}$ in Eq. (52) in [64, providing an additional check of our results.

Note that the expression (4.25) for $\Psi$ results from the Newtonian potential $U_{\mathrm{E}}$ if we consider the expression (1.9) for the "effective" gravitational constant as seen in the local system.

2) Equating the external terms of order $|\mathbf{X}|^{0}$ give us the equation

$$
\dot{B}(t)=-\frac{1}{8} v_{E}^{4}+2(\gamma+1) v_{E}^{i} \bar{w}^{i}\left(\mathbf{x}_{E}\right)-\left(\gamma+\frac{1}{2}\right) v_{E}^{2} \bar{w}\left(\mathbf{x}_{E}\right)+\left(\beta-\frac{1}{2}\right) \bar{w}^{2}\left(\mathbf{x}_{E}\right) .
$$

3) Matching the terms of order $|\mathbf{X}|^{1}$ leads to the equations of motion of the origin of the local system with respect to the barycentric PPN reference system

$$
\begin{gathered}
a_{E}^{i}=\bar{w}_{, i}\left(\mathbf{x}_{E}\right)-R_{j}^{a} Q_{a}\left(\delta^{i j}-\frac{1}{c^{2}}\left(v_{E}^{2} \delta^{i j}+(2+\gamma) \bar{w}\left(\mathbf{x}_{E}\right) \delta^{i j}+\frac{1}{2} v_{E}^{i} v_{E}^{j}\right)\right) \\
+\frac{1}{c^{2}}\left(2(1+\gamma) \dot{\bar{w}}^{i}\left(\mathbf{x}_{E}\right)+\left(\gamma v_{E}^{2}-2(\gamma+\beta) \bar{w}\left(\mathbf{x}_{E}\right)\right) \bar{w}_{, i}\left(\mathbf{x}_{E}\right)-(2 \gamma+1) v_{E}^{i} \dot{\bar{w}}\left(\mathbf{x}_{E}\right)\right. \\
\left.-2(1+\gamma) v_{E}^{j} \bar{w}_{, i}^{j}\left(\mathbf{x}_{E}\right)-v_{E}^{i} v_{E}^{j} \bar{w}_{, j}\left(\mathbf{x}_{E}\right)\right)+\mathcal{O}\left(c^{-4}\right)
\end{gathered}
$$

Note that the Newtonian terms $\bar{w}_{, i}\left(\mathbf{x}_{E}\right)$ and $Q_{a}$ in (4.28) should be computed at moments of time $t$ and $T$ related to each other by $T=t-1 / c^{2} A(t)+\mathcal{O}\left(c^{-4}\right)$, that is by the coordinate transformation (3.44) evaluated at $\mathbf{x}=\mathbf{x}_{E}$. For $Q_{a}=0$, (4.28) coincides with the PPN equations of motion of a test particle (geodetic equation), provided that the gravitational potentials of the local subsystem $w_{\mathrm{E}}, w_{\mathrm{E}}^{i}$ are excluded from the metric.

We consider $Q_{a}$ as a known function of $T$ specified in the local reference system $\left(T, X^{a}\right)$ with post-Newtonian accuracy. When computing the Newtonian terms in (1.28) with postNewtonian accuracy, $Q_{a}$ should be expressed in terms of global coordinates. It is easy to see that all relativistic terms in (4.28) proportional to $Q_{a}$ are due to the re-computation of $Q_{a}$ from the local reference system to the global one. Indeed, if a test particle is situated 
at the origin of the local reference system $\left(X_{\text {test }}^{a}=0\right)$ and if its local coordinate velocity is equal to zero $\left(d X_{\text {test }}^{a} / d T=0\right)$ then making use of (3.44)-(3.45) we get

$$
\begin{aligned}
\frac{d^{2} x_{\text {test }}^{i}}{d t^{2}}-a_{E}^{i}= & R_{j}^{a} \frac{d^{2} X_{\text {test }}^{a}}{d T^{2}}\left(\delta^{i j}-\frac{1}{c^{2}}\left(v_{E}^{2} \delta^{i j}+(2+\gamma) \bar{w}\left(\mathbf{x}_{E}\right) \delta^{i j}+\frac{1}{2} v_{E}^{i} v_{E}^{j}\right)\right) \\
& +\mathcal{O}\left(c^{-4}\right),
\end{aligned}
$$

where $d^{2} x_{\text {test }}^{i} / d t^{2}-a_{E}^{i}$ is the global coordinate acceleration of the test particle relative to the origin of the local reference system. The terms on the right-hand side of (4.29) exactly match those in (4.28) that are proportional to $Q_{a} \cdot Q_{a}$ is, thus, the acceleration of the instantaneous locally inertial reference system (whose origin coincides with that of the local reference system at a given moment of time) expressed in the local reference system. This also can be seen from the equations of motion of test particles relative to the local reference system (see, Section $\nabla \Pi 1$ below). The equations of motion can be represented as

$$
\ddot{X}_{\text {test }}^{a}=Q^{a}(T)+f^{a}\left(\mathbf{X}_{\text {test }}, \dot{\mathbf{X}}_{\text {test }}\right)+\mathcal{O}\left(c^{-4}\right),
$$

where $f^{a}\left(\mathbf{X}_{\text {test }}, \dot{\mathbf{X}}_{\text {test }}\right)$ is some force depending on position and/or velocity of the particle (i.e., the only term in the equations of motion independent on the actual trajectory of the particle is $\left.Q^{a}\right)$. Note also that $Q_{a}$ can be directly related to the 4-acceleration of the worldline of the origin of the local reference system (see, e.g., [22]).

4) Matching of the external terms of order $|\mathbf{X}|^{l}, l \geq 2$ in closed form leads to an expression for the relativistic tidal potential of the form

$$
\begin{aligned}
& W_{\mathrm{T}}(T, \mathbf{X})= \bar{w}(t, \mathbf{x})-\bar{w}\left(\mathbf{x}_{E}\right)-\bar{w}_{, j}\left(\mathbf{x}_{E}\right) r_{E}^{j} \\
&+\frac{1}{c^{2}}(-2(1+\gamma) v_{E}^{i}\left(\bar{w}^{i}(t, \mathbf{x})-\bar{w}^{i}\left(\mathbf{x}_{E}\right)-\bar{w}_{, j}^{i}\left(\mathbf{x}_{E}\right) r_{E}^{j}\right)+(1+\gamma) v_{E}^{2} W_{\mathrm{T}} \\
&+(1+\gamma) \dot{w}^{i, j}\left(\mathbf{x}_{E}\right) r_{E}^{i} r_{E}^{j}+\frac{1}{2} \gamma \ddot{\bar{w}}\left(\mathbf{x}_{E}\right) r_{E}^{2}+\left(\frac{1}{2}-\beta-\gamma\right)\left(a_{E}^{i} r_{E}^{i}\right)^{2} \\
&+(1-2 \beta-2 \gamma) Q_{a} X^{a} a_{E}^{i} r_{E}^{i}-\gamma v_{E}^{i} r_{E}^{i} \dot{\bar{w}}_{, j}\left(\mathbf{x}_{E}\right) r_{E}^{j}+\frac{1}{2} \gamma r_{E}^{2} R_{i}^{a} a_{E}^{i} Q_{a} \\
&\left.+C_{, T}(T, \mathbf{X})+2(1-\beta)\left(\bar{w}\left(\mathbf{x}_{E}\right)+a_{E}^{i} r_{E}^{i}\right) W_{\mathrm{T}}\right)+\mathcal{O}\left(c^{-4}\right) \\
& W_{\mathrm{T}}^{a}(T, \mathbf{X})=R_{i}^{a}\left\{\bar{w}^{i}(t, \mathbf{x})-\bar{w}^{i}\left(\mathbf{x}_{E}\right)-\bar{w}_{, j}^{i}\left(\mathbf{x}_{E}\right) r_{E}^{j}-v_{E}^{i} W_{\mathrm{T}}(T, \mathbf{X})\right. \\
&\left.\quad+\frac{1}{2(1+\gamma)}\left(\gamma\left(r_{E}^{i} \dot{a}_{E}^{j} r_{E}^{j}-\frac{1}{2} \dot{a}_{E}^{i} r_{E}^{2}\right)-C_{, i}(t, \mathbf{x})\right)\right\}+\mathcal{O}\left(c^{-2}\right)
\end{aligned}
$$


where $C_{, T}$ is a partial derivative of $C$ with respect to the local coordinate time $T$. E.g., for

$$
C(T, \mathbf{X})=C_{1}(T, \mathbf{X})=\frac{\gamma-2}{10} \mathbf{X}^{2} \dot{a}_{E}^{i} R_{i}^{a} X^{a}
$$

one gets

$$
C_{, T}(T, \mathbf{X})=\frac{\gamma-2}{10} \mathbf{X}^{2} \ddot{a}_{E}^{i} R_{i}^{a} X^{a}
$$

Let us note that in the Newtonian terms in the right-hand side (4.31) the moment of time $t$ depends on both $T$ and $\mathbf{X}$ according to (3.44)-(3.45). This should be accounted for when computing, e.g., partial derivatives of $W_{\mathrm{T}}$ with respect to $T$.

From (4.31) it follows that

$$
\begin{aligned}
c^{2} W_{\mathrm{T}, a a}= & \ddot{Q}_{a} X^{a}+W_{\mathrm{T}, T T}-\eta\left(Q_{a}+W_{\mathrm{T}, a}\right) R_{i}^{a} a_{E}^{i} \\
& +(\gamma-1)\left(\ddot{\bar{w}}\left(\mathbf{x}_{E}\right)+R_{i}^{a}{ }_{i} \ddot{a}_{E}^{i} X^{a}\right)-2(\beta-1) a_{E}^{2}+\mathcal{O}\left(c^{-2}\right), \\
W_{\mathrm{T}, a a b b}= & \mathcal{O}\left(c^{-4}\right) .
\end{aligned}
$$

Eqs. (4.35) and (4.19) can be considered as effective field equations for the external potentials in local coordinates.

We have derived all unknown functions with post-Newtonian accuracy and thereby obtained the local PPN metric tensor as well as the transformations between the local and global PPN reference systems.

\section{E. Energy-momentum tensor in the local reference system}

It is interesting to check the form of the energy-momentum tensor for an ideal fluid (3.19) in local coordinates, $\mathcal{T}^{\alpha \beta}$. The energy-momentum tensor $T^{\alpha \beta}$ in the global PPN reference system is related to $\mathcal{T}^{\alpha \beta}$ by

$$
\mathcal{T}^{\alpha \beta}(T, \mathbf{X})=\frac{\partial X^{\alpha}}{\partial x^{\mu}} \frac{\partial X^{\beta}}{\partial x^{\nu}} T^{\mu \nu}(t, \mathbf{x})
$$


For an ideal fluid $T^{\mu \nu}$ is defined by $(3.19)$ or (3.22). Substituting the transformation rules (3.44)-(3.45) and the expressions (4.3), (4.6) and (4.7) for $A, D^{i j}$ and $D^{i j k}$ respectively one gets

$$
\begin{aligned}
& \mathcal{T}^{00}=\rho c^{2}\left[1+\frac{1}{c^{2}}\left(V^{2}+\Pi+2 W\right)\right]+\mathcal{O}\left(c^{-2}\right), \\
& \mathcal{T}^{0 a}=\rho c V^{a}\left[1+\frac{1}{c^{2}}\left(V^{2}+\Pi+2 W\right)\right]+\frac{1}{c} p V^{a}+\mathcal{O}\left(c^{-3}\right), \\
& \mathcal{T}^{a b}=\rho V^{a} V^{b}+\delta^{a b} p+\mathcal{O}\left(c^{-2}\right),
\end{aligned}
$$

where $V^{a}$ is the velocity of matter relative to the local reference system, $\rho(T, \mathbf{X})=\rho(t, \mathbf{x})$ is the invariant density, $\Pi(T, \mathbf{X})=\Pi(t, \mathbf{x})$ is the specific energy density, and $p(T, \mathbf{X})=p(t, \mathbf{x})$ is the isotropic pressure, $W(T, \mathbf{X})=w(t, \mathbf{x})-\bar{w}\left(\mathbf{x}_{E}\right)-a_{E}^{i} r_{E}^{i}+\mathcal{O}\left(c^{-2}\right)$. One can also rewrite (4.38) as

$$
\begin{aligned}
\mathcal{T}^{00} & =\hat{\rho}^{*} c^{2}\left[1+\frac{1}{c^{2}}\left(\frac{1}{2} V^{2}+\Pi-(3 \gamma-2) W\right)\right]+\mathcal{O}\left(c^{-2}\right), \\
\mathcal{T}^{0 a} & =\hat{\rho}^{*} c V^{a}\left[1+\frac{1}{c^{2}}\left(\frac{1}{2} V^{2}+\Pi-(3 \gamma-2) W\right)\right]+\frac{1}{c} p V^{a}+\mathcal{O}\left(c^{-3}\right), \\
\mathcal{T}^{a b} & =\hat{\rho}^{*} V^{a} V^{b}+\delta^{a b} p+\mathcal{O}\left(c^{-2}\right),
\end{aligned}
$$

where

$$
\hat{\rho}^{*}(T, \mathbf{X})=\rho(T, \mathbf{X})(-\mathcal{G})^{1 / 2} \frac{c d T}{d s}=\rho\left[1+\frac{1}{c^{2}}\left(\frac{1}{2} V^{2}+3 \gamma W\right)+\mathcal{O}\left(c^{-4}\right)\right]
$$

so that

$$
\frac{\partial}{\partial T} \hat{\rho}^{*}+\frac{\partial}{\partial X^{a}}\left(\hat{\rho}^{*} V^{a}\right)=\mathcal{O}\left(c^{-4}\right)
$$

Thus, the functional form of the energy momentum tensor is the same in the global and local PPN reference systems.

\section{OTHER FORMS OF THE LOCAL METRIC}

In the previous Section we have succeeded to construct a local reference system $\left(T, X^{a}\right)$ which satisfies property A from Section [, but does not satisfy property $\mathbf{B}$. It is interesting to 
check if there exist other versions of a local reference system satisfying properties $\mathbf{A}$ and/or B. To this end let us consider another set of local coordinates $\left(\tilde{T}, \tilde{X}^{a}\right)$ related to $\left(T, X^{a}\right)$ by the following transformations

$$
\begin{aligned}
\tilde{T} & =T \\
\tilde{X}^{a} & =X^{a}+\frac{1}{c^{2}} \frac{\eta}{\gamma}\left(R_{i}^{a} R_{j}^{b} D^{i j} X^{b}+R_{i}^{a} R_{j}^{b} R_{k}^{c} D^{i j k} X^{b} X^{c}\right)+\mathcal{O}\left(c^{-4}\right),
\end{aligned}
$$

where $D^{i j}$ and $D^{i j k}$ are defined by 4.6$)-(4.7)$. One can show that in $\left(\tilde{T}, \tilde{X}^{a}\right)$ the metric tensor reads

$$
\begin{gathered}
\tilde{G}_{00}=-1+\frac{2}{c^{2}} \tilde{W}(\tilde{T}, \tilde{\mathbf{X}})-\frac{2}{c^{4}} \beta \tilde{W}^{2}(\tilde{T}, \tilde{\mathbf{X}})+\mathcal{O}\left(c^{-5}\right) \\
\tilde{G}_{0 a}=-\frac{2(1+\gamma)}{c^{3}} \tilde{W}^{a}(\tilde{T}, \tilde{\mathbf{X}})-\frac{1}{c^{3}} \eta\left(\dot{\bar{w}}\left(\mathbf{x}_{E}\right) \tilde{X}^{a}+\tilde{X}^{a} R^{b}{ }_{i} \dot{a}_{E}^{i} \tilde{X}^{b}-\frac{1}{2} R_{i}^{a} \dot{a}_{E}^{i} \tilde{\mathbf{X}}^{2}\right)+\mathcal{O}\left(c^{-5}\right) \\
\tilde{G}_{a b}=\delta_{a b}\left(1+\frac{2}{c^{2}}\left(\gamma \tilde{W}(\tilde{T}, \tilde{\mathbf{X}})-\eta\left(\bar{w}\left(\mathbf{x}_{E}\right)+R_{i}^{a} a_{E}^{i} \tilde{X}^{a}\right)\right)\right)+\mathcal{O}\left(c^{-4}\right) \\
\tilde{W}(\tilde{T}, \tilde{\mathbf{X}})=\tilde{W}_{\mathrm{E}}(\tilde{T}, \tilde{\mathbf{X}})+\tilde{Q}_{a}(\tilde{T}) \tilde{X}^{a}+\tilde{W}_{\mathrm{T}}(\tilde{T}, \tilde{\mathbf{X}}) \\
\tilde{W}^{a}(\tilde{T}, \tilde{\mathbf{X}})=\tilde{W}_{\mathrm{E}}^{a}(\tilde{T}, \tilde{\mathbf{X}})+\frac{1}{2} \varepsilon_{a b c} \tilde{C}_{b}(\tilde{T}) \tilde{X}^{c}+\tilde{W}_{\mathrm{T}}^{a}(\tilde{T}, \tilde{\mathbf{X}})
\end{gathered}
$$

where

$$
\begin{aligned}
& \tilde{W}_{\mathrm{E}}= G \int_{V} \tilde{\Sigma}\left(\tilde{T}, \tilde{\mathbf{X}}^{\prime}\right) \frac{1}{\left|\tilde{\mathbf{X}}-\tilde{\mathbf{X}}^{\prime}\right|} d^{3} \tilde{X}^{\prime} \\
&+\frac{1}{2 c^{2}} G \frac{\partial^{2}}{\partial \tilde{T}^{2}} \int_{V} \tilde{\Sigma}\left(\tilde{T}, \tilde{\mathbf{X}}^{\prime}\right)\left|\tilde{\mathbf{X}}-\tilde{\mathbf{X}}^{\prime}\right| d^{3} \tilde{X}^{\prime}+\mathcal{O}\left(c^{-4}\right), \\
& \tilde{W}_{\mathrm{E}}^{a}= G \int_{V} \tilde{\Sigma}^{a}\left(\tilde{T}, \tilde{\mathbf{X}}^{\prime}\right) \frac{1}{\left|\tilde{\mathbf{X}}-\tilde{\mathbf{X}}^{\prime}\right|} d^{3} \tilde{X}^{\prime}+\mathcal{O}\left(c^{-2}\right), \\
& \tilde{\Sigma}=\frac{1}{c^{2}}\left(\tilde{\mathcal{T}}^{00}+\gamma \tilde{\mathcal{T}}^{a a}+\frac{1}{c^{2}} \tilde{\mathcal{T}}^{00}(3 \gamma-2 \beta-1) \tilde{W}\right)+\mathcal{O}\left(c^{-4}\right), \\
& \tilde{\Sigma}^{a}=\frac{1}{c} \tilde{\mathcal{T}}^{0 a}+\mathcal{O}\left(c^{-2}\right),
\end{aligned}
$$

where $\tilde{\mathcal{T}}^{\mu \nu}$ are the components of the energy-momentum tensor in coordinates $(\tilde{T}, \tilde{\mathbf{X}})$, and $\tilde{W}_{\mathrm{T}}$ and $\tilde{W}_{\mathrm{T}}^{a}$ are some external tidal $\left(\sim \mathcal{O}\left(\tilde{\mathbf{X}}^{2}\right)\right)$ potentials.

The internal potentials $\tilde{W}_{E}$ and $\tilde{W}_{E}^{a}$ have the same form as the potentials of the corresponding isolated source provided that the tidal influence of external metric is neglected 
and, therefore, in coordinates $(\tilde{T}, \tilde{\mathbf{X}})$ property B from Section 『 is satisfied. However, the terms proportional to $\eta$ in $\tilde{G}_{0 a}$ and $\tilde{G}_{a b}$ represent non-tidal external potentials, thus violating property A.

Although any reference system can be obviously used, we believe that the original local coordinates $(T, \mathbf{X})$ are more convenient since a violation of the Strong Equivalence Principle is presented solely by the $\Psi$ term in $G_{00}$. Moreover, the appearance of non-tidal external potentials in $\tilde{G}_{0 a}$ and $\tilde{G}_{a b}$ results in a more complicated interpretation of observational data in $(\tilde{T}, \tilde{\mathbf{X}})$ (e.g., involving a complicated redefinition of locally measured physical constants and parameters). In the following we use a local reference system with coordinates $(T, \mathbf{X})$.

\section{MULTIPOLE EXPANSIONS OF THE INTERNAL POTENTIALS}

Although the metric tensor of the local PPN reference system described above enables us to discuss any physical phenomena in this reference system in the first post-Newtonian approximation, for many reasons an expansion of the gravitational potentials in terms of multipole moments is desirable. The main usefulness of mass (and spin) multipole moments of a body results from the fact that for astronomical, almost 'spheroidal' bodies usually only a few determine the equations of motion to sufficient accuracy. Numerical values of multipole moments can be obtained directly from various astronomical observations without evaluating volume integrals like (3.35) appearing in the local metric tensor.

The complete solution of the problem of multipole expansions of gravitational potentials and equations of motion has been found in [22,27,28] for the case of General Relativity. The authors use the so called Blanchet-Damour (BD) mass multipole moments 66, 69. The BD moments are defined physically by reference to multipole moments which can be read off from gravitational waves emitted by an isolated system of matter. The principal attractive

property of the $\mathrm{BD}$ moments for their practical applications in multipole expansions is contained in Theorem 6 of [22]. The Theorem states that the multipole expansions of general-relativistic post-Newtonian gravitational potentials in terms of the BD multipole 
moments are almost of Newtonian form [22]. In [22,27,28] it has been shown that this almost Newtonian form of multipole expansions is advantageous for both translational and rotational equations of motion.

It turns out that in the PPN formalism we can find adequate multipole moments by "mimicking" the almost Newtonian form of multipole expansion of the post-Newtonian gravitational potential. It is easy to see that with the following definition of generalized BD moments

$$
\begin{aligned}
\mathcal{M}_{L}= & \int_{V} \Sigma \hat{X}^{L} d^{3} X+\frac{1}{2(2 l+3)} \frac{1}{c^{2}} \frac{d^{2}}{d T^{2}} \int_{V} \Sigma \hat{X}^{L} X^{2} d^{3} X \\
& -\frac{2(\gamma+1)(2 l+1)}{(l+1)(2 l+3)} \frac{1}{c^{2}} \frac{d}{d T} \int_{V} \Sigma^{a} \hat{X}^{a L} d^{3} X, \quad l \geq 0, \\
\mathcal{S}_{L}= & \int_{V} \varepsilon^{b c\left\langle a_{l}\right.} \hat{X}^{L-1\rangle b} \Sigma^{c} d^{3} X, \quad l \geq 1,
\end{aligned}
$$

one gets

$$
\begin{aligned}
W_{E}=G \sum_{l=0}^{\infty} \frac{(-1)^{l}}{l !}\left[\mathcal{M}_{L} \partial_{L} \frac{1}{|\mathbf{X}|}+\frac{1}{2 c^{2}} \ddot{\mathcal{M}}_{L} \partial_{L}|\mathbf{X}|\right]+\frac{2(1+\gamma)}{c^{2}} \Lambda_{, T}+\mathcal{O}\left(c^{-4}\right), \\
2(1+\gamma) W_{E}^{a}=-2(1+\gamma) G \sum_{l=1}^{\infty} \frac{(-1)^{l}}{l !}\left[\dot{\mathcal{M}}_{a L-1} \partial_{L-1} \frac{1}{|\mathbf{X}|}+\frac{l}{l+1} \varepsilon_{a b c} \mathcal{S}_{c L-1} \partial_{b L-1} \frac{1}{|\mathbf{X}|}\right] \\
-2(1+\gamma) \Lambda_{, a}+\mathcal{O}\left(c^{-2}\right),
\end{aligned}
$$

where

$$
\begin{gathered}
\Lambda=G \sum_{l=0}^{\infty} \frac{(-1)^{l}}{(l+1) !} \frac{2 l+1}{2 l+3} \mathcal{P}_{L} \partial_{L} \frac{1}{|\mathbf{X}|} \\
\mathcal{P}_{L}=\int_{V} \Sigma^{a} \hat{X}^{a L} d^{3} X .
\end{gathered}
$$

The terms containing $\Lambda$ can be eliminated from (6.3)-(6.4) by a transformation of time coordinate

$$
T^{\prime}=T-\frac{2(1+\gamma)}{c^{4}} \Lambda
$$

This gauge is called skeletonized harmonic gauge in [22] (no $\Lambda$-terms appear in (6.3)-(6.4) and the gravitational potentials $W_{\mathrm{E}}$ and $W_{\mathrm{E}}^{a}$ are "skeletonized" by the moments $\mathcal{M}_{L}$ and 
$\left.\mathcal{S}_{L}\right)$. The generalized BD multipole moments (6.1)- -6.2 obviously do not possess all nice properties of the BD moments in General Relativity. However, the expansion (6.3) has the same almost Newtonian form as the corresponding expansion in General Relativity. This fosters the hope that the generalized BD multipole moments are suitable for the use in multipole expanded PPN equations of motion.

\section{TIDAL EXPANSIONS OF THE EXTERNAL POTENTIALS}

Although we have derived the local external potentials $W_{\mathrm{T}}$ and $W_{\mathrm{T}}^{i}$ in closed form, in many cases it is preferable to have tidal expansions of them. These expansions are very convenient, e.g., for a discussion of equations of motions in the local reference system. Tidal expansions of the external potentials entering $G_{00}$ and $G_{0 i}$ are quite straightforward to derive and read

$$
\begin{aligned}
& Q_{a} X^{a}+W_{\mathrm{T}}=\sum_{l=1}^{\infty} \frac{1}{l !} Q_{L} \hat{X}^{L}-\frac{1}{c^{2}} \sum_{l=3}^{\infty} \frac{1}{l !}\left(\dot{R}_{L}-\dot{V}_{L}\right) \hat{X}^{L}+\frac{1}{2 c^{2}} \sum_{l=0}^{\infty} \frac{1}{l !} \frac{X^{2}}{2 l+3} K_{L} \hat{X}^{L}, \\
& 2(1+\gamma) W_{\mathrm{T}}^{a}=2(1+\gamma) \sum_{l=2}^{\infty} \frac{l}{(l+1) !} \varepsilon_{a b c_{l}} C_{b L-1} \hat{X}^{L} \\
&-2(1+\gamma) \sum_{l=1}^{\infty} \frac{1}{(l+1) !} \frac{2 l+1}{2 l+3} \dot{Q}_{L} \hat{X}^{a L} \\
&+\sum_{l=2}^{\infty} \frac{1}{l !}\left(R_{a L}-V_{a L}\right) \hat{X}^{L} \\
&+\frac{1}{5}(\gamma-1)\left(R_{i}^{b} \dot{a}_{E}^{i} X^{a} X^{b}-2 R_{i}^{a} \dot{a}_{E}^{i} X^{2}\right)+\mathcal{O}\left(c^{-2}\right),
\end{aligned}
$$

where the symmetric tracefree tidal moments $Q_{L}, C_{L}, K_{L}$ and $R_{L}$ are defined as

$$
\begin{aligned}
Q_{L}=\operatorname{STF}_{L}\{ & d_{L} \bar{w}\left(\mathbf{x}_{E}\right)+\frac{1}{c^{2}}\left(2(1+\gamma) R_{i}^{a_{l}} d_{L-1} \dot{\bar{w}}^{i}\left(\mathbf{x}_{E}\right)-2(1+\gamma) v_{E}^{i} d_{L} \bar{w}^{i}\left(\mathbf{x}_{E}\right)\right. \\
& +(1+\gamma) v_{E}^{2} d_{L} \bar{w}\left(\mathbf{x}_{E}\right)-\frac{1}{2} l R_{i}^{a_{l}} R_{j}^{b} v_{E}^{i} v_{E}^{j} d_{b L-1} \bar{w}\left(\mathbf{x}_{E}\right)+\lambda^{L} \\
& -[\gamma l+2(\beta-1)] \bar{w}\left(\mathbf{x}_{E}\right) d_{L} \bar{w}\left(\mathbf{x}_{E}\right)+[l-2(1+\gamma)] R_{i}^{a_{l}} v_{E}^{i} d_{L-1} \dot{\bar{w}}\left(\mathbf{x}_{E}\right) \\
& \left.\left.-[\gamma l(l-1)+2(\beta-1) l+2(1+\gamma)] R_{i}^{a_{l}} a_{E}^{i} d_{L-1} \bar{w}\left(\mathbf{x}_{E}\right)\right)\right\} \\
& +\mathcal{O}\left(c^{-4}\right), \quad l \geq 2,
\end{aligned}
$$




$$
\begin{gathered}
\lambda^{a b}=(2 \beta+2 \gamma-1) R_{i}^{a} R_{j}^{b} a_{E}^{i} a_{E}^{j}, \\
\lambda^{L}=0, \quad l \geq 3, \\
R_{L}=2(1+\gamma) \mathrm{STF}_{L}\left\{R_{i}^{a_{1}}\left[d_{L-1} \bar{w}^{i}\left(\mathbf{x}_{E}\right)-v_{E}^{i} d_{L-1} \bar{w}\left(\mathbf{x}_{E}\right)\right]\right\}+\mathcal{O}\left(c^{-2}\right), \quad l \geq 3, \\
K=(\gamma-1) \ddot{\bar{U}}\left(\mathbf{x}_{E}\right)+2(1-\beta) a_{E}^{2}-\eta Q_{a} R_{i}^{a} a_{E}^{i}+\mathcal{O}\left(c^{-2}\right), \\
K_{a}=\ddot{Q}_{a}-\eta Q_{a b} R_{i}^{b} a_{E}^{i}+(\gamma-1) R_{i}^{a} \ddot{a}_{E}^{i}+\mathcal{O}\left(c^{-2}\right), \\
K_{L}=\ddot{Q}_{L}-\eta Q_{a L} R_{i}^{a} a_{E}^{i}+\mathcal{O}\left(c^{-2}\right), \quad l \geq 2, \\
C_{L}=\operatorname{STF}_{L}\left\{\varepsilon_{a_{l} b c} R_{i}^{c} d_{b L-1}\left(\bar{w}^{i}\left(\mathbf{x}_{E}\right)-v_{E}^{i} \bar{w}\left(\mathbf{x}_{E}\right)\right)\right\}, l \geq 2,
\end{gathered}
$$

(note that the expression in braces is already trace free). Here $d_{L}$ is the shorthand notation

$$
d_{L} \Phi(t, \mathbf{x}) \equiv d_{a_{1} \ldots a_{l}} \Phi(t, \mathbf{x}) \equiv R_{i_{1}}^{a_{1}} \ldots R_{i_{l}}^{a_{l}} \frac{\partial^{l}}{\partial x^{i_{1}} \ldots \partial x^{i_{l}}} \Phi(t, \mathbf{x})
$$

and $d_{L} \Phi\left(\mathbf{x}_{E}\right)$ should be understood as the corresponding derivative evaluated at $\mathbf{x}=\mathbf{x}_{E}$. In order to compute $Q_{L}$ for some given moment of local time $T$ with the aid of (7.3) one has to evaluate $d_{L} \bar{w}\left(\mathbf{x}_{E}\right)$ at moment of global time $t$ related with $T$ as $T=t-1 / c^{2} A(t)+\mathcal{O}\left(c^{-4}\right)$, that is by the transformations (3.44) evaluated at $\mathbf{x}=\mathbf{x}_{E}$.

Expansion (7.1) with (7.3)-(7.6) can be derived by differentiating (4.31) and using (4.35)(4.36) and the fact that for any $S$

$$
\begin{aligned}
S= & \left.\sum_{l=1}^{\infty} \frac{1}{l !} \frac{\partial^{l}}{\partial X^{a_{1}} \ldots X^{a_{l}}} S\right|_{\mathbf{X}=0} \hat{X}^{L} \\
& +\left.\frac{1}{l !} \frac{1}{2(2 l+3)} \frac{\partial^{l}}{\partial X^{a_{1}} \ldots X^{a_{l}}} S_{, b b}\right|_{\mathbf{X}=0} \hat{X}^{L} \mathbf{X}^{2}+\mathcal{O}\left(S_{, b b c c}\right) .
\end{aligned}
$$

The moments $K_{L}$ represent the multipole expansion of 4.35): $c^{-2} K_{L}=W_{\mathrm{T}, a a L}$. The quantities $V_{L}$ are arbitrary STF moments appearing in the multipole expansion (4.15)(4.17) of the function $C(T, \mathbf{X})$. One can get rid of the moments $R_{L}$ in $(7.1)-(7.2)$ by setting $V_{L}=R_{L}$, that is, by choosing a special solution for $C(T, \mathbf{X})$

$$
C(T, \mathbf{X})=\frac{\gamma-2}{10} X^{2} R_{i}^{a} \dot{a}_{E}^{i} X^{a}+\sum_{l=3}^{\infty} \frac{1}{l !} R_{L} \hat{X}^{L} .
$$


In the limit of General Relativity $\beta=\gamma=1$ only the two families $Q_{L}$ and $C_{L}$ play a role in $(7.1)-(7.2)$. These are related to the tidal moments $G_{L}$ and $H_{L}$ of the DSX formalism 22] as

$$
\begin{aligned}
& G_{L}=\left.Q_{L}\right|_{\beta=\gamma=1}, \\
& H_{L}=-\left.4 C_{L}\right|_{\beta=\gamma=1} .
\end{aligned}
$$

Because of the gauge dependence the family $R_{L}$ does not appear, e.g., in the equations of motion of test particles and $N$ extended bodies which we derive in the next Sections. This can be shown also by computing the tidal expansions of the following quantities which directly enter the post-Newtonian equations of motion and are independent of the gauge of the time coordinate

$$
\begin{aligned}
Q^{a}+ & W_{\mathrm{T}, a}+\frac{1}{c^{2}} 2(1+\gamma) W_{\mathrm{T}, T}^{a}= \\
& \sum_{l=0}^{\infty} \frac{1}{l !} Q_{a L} \hat{X}^{L}+\frac{1}{c^{2}} 2(1+\gamma) \sum_{l=2}^{\infty} \frac{1}{l !} \frac{l}{l+1} \varepsilon_{a b c} \dot{C}_{b L-1} \hat{X}^{c L-1} \\
& +\frac{1}{c^{2}} \sum_{l=0}^{\infty} \frac{1}{l !} \frac{1}{2(2 l+3)} \ddot{Q}_{a L} \mathbf{X}^{2} \hat{X}^{L}-\frac{1}{c^{2}} \sum_{l=2}^{\infty} \frac{1}{l !} \frac{(4 \gamma+3) l-2(1+\gamma)}{2 l+1} \ddot{Q}_{L-1} \hat{X}^{a L-1} \\
& +\frac{1}{3 c^{2}} X^{a}\left((\gamma-1) \ddot{w}\left(\mathbf{x}_{E}\right)+2(1-\beta) a_{E}^{2}\right)-\frac{1}{6 c^{2}}(\gamma-1) R_{i}^{a} \ddot{a}_{E}^{i} \mathbf{X}^{2} \\
& +\frac{2}{5 c^{2}}(\gamma-1) R_{i}^{b} \ddot{a}_{E}^{i} \hat{X}^{a b}-\frac{1}{c^{2}} \eta \sum_{l=0}^{\infty} \frac{1}{l !} \frac{1}{2 l+3} R_{i}^{b} a_{E}^{i} Q_{b L} \hat{X}^{a L} \\
& -\frac{1}{2 c^{2}} \eta \sum_{l=0}^{\infty} \frac{1}{l !} \frac{1}{2 l+3} R_{i}^{b} a_{E}^{i} Q_{a b L} \mathbf{X}^{2} \hat{X}^{L}+\mathcal{O}\left(c^{-4}\right), \\
- & 2(1+\gamma) \varepsilon_{a b c} W_{\mathrm{T}, c}^{b}=2(1+\gamma) \sum_{l=1}^{\infty} \frac{1}{l !}\left(C_{a L} \hat{X}^{L}+\frac{l}{l+1} \varepsilon_{a b c} \hat{X}^{b L-1} \dot{Q}_{c L-1}\right) \\
& +(\gamma-1) \varepsilon_{a b c} R_{i}^{b} \dot{a}_{E}^{i} X^{c}+\mathcal{O}\left(c^{-2}\right) .
\end{aligned}
$$

In the limit of General Relativity (7.15) and (7.16) coincide with Equation (6.23a) and (6.23b) of [22], respectively. 


\section{TRANSLATIONAL EQUATIONS OF MOTION OF A TEST PARTICLE IN THE LOCAL REFERENCE SYSTEM}

\section{A. The equations of motion in closed form}

It is well know that a test particle moves along a geodetic of spacetime as determined by the metric tensor. The geodetic equation in the metric (3.32) reads

$$
\begin{gathered}
\ddot{X}_{\mathrm{s}}^{a}=W_{, a}+\frac{1}{c^{2}}\left\{2(1+\gamma) W_{, T}^{a}-2(\beta+\gamma) W W_{, a}-(2 \gamma+1) W_{, T} \dot{X}_{\mathrm{s}}^{a}-2(1+\gamma) W_{, b} \dot{X}_{\mathrm{s}}^{b} \dot{X}_{\mathrm{s}}^{a}\right. \\
\left.+\gamma W_{, a} \dot{X}_{\mathrm{s}}^{b} \dot{X}_{\mathrm{s}}^{b}+2(1+\gamma)\left(W_{, b}^{a}-W_{, a}^{b}\right) \dot{X}_{\mathrm{s}}^{b}\right\}+\mathcal{O}\left(c^{-4}\right)
\end{gathered}
$$

Here, $\mathbf{X}_{\mathrm{s}}$ are local coordinates of the test particle (e.g., a satellite), and all potentials and their derivatives should be evaluated at $(T, \mathbf{X})=\left(T, \mathbf{X}_{\mathbf{s}}(T)\right)$. Accounting for the split (3.33)- (3.34) of the local potentials entering into (3.32) it can be seen that the equations of motion in the local reference system read

$$
\ddot{X}_{\mathrm{s}}^{a}=\Phi_{\mathrm{E}}^{a}+\Phi_{\mathrm{el}}^{a}+\frac{1}{c^{2}}\left(\Phi_{\mathrm{coup}}^{a}+\Phi_{\mathrm{mg}}^{a}+\Phi_{\Psi}^{a}\right)+\mathcal{O}\left(c^{-4}\right)
$$

where $\Phi_{\mathrm{E}}^{a}$ are the terms describing the Earth's gravitational field in the absence of an external world

$$
\begin{aligned}
\Phi_{\mathrm{E}}^{a}=W_{\mathrm{E}, a}+\frac{1}{c^{2}} & \left(2(1+\gamma) W_{\mathrm{E}, T}^{a}-2(\gamma+\beta) W_{\mathrm{E}} W_{\mathrm{E}, a}\right. \\
& -(2 \gamma+1) W_{\mathrm{E}, T} \dot{X}_{\mathrm{s}}^{a}-2(1+\gamma) W_{\mathrm{E}, b} \dot{X}_{\mathrm{s}}^{b} \dot{X}_{\mathrm{s}}^{a}+\gamma W_{\mathrm{E}, a} \dot{X}_{\mathrm{s}}^{b} \dot{X}_{\mathrm{s}}^{b} \\
& \left.+2(1+\gamma) \dot{X}_{\mathrm{s}}^{b}\left(W_{\mathrm{E}, b}^{a}-W_{\mathrm{E}, a}^{b}\right)\right)+\mathcal{O}\left(c^{-4}\right),
\end{aligned}
$$

The Earth-third body coupling term $\Phi_{\text {coup }}^{a}$ due to the only nonlinear term in (8.1) reads

$$
\Phi_{\text {coup }}^{a}=-2(\gamma+\beta)\left(W_{\mathrm{E}}\left(Q_{a}+W_{\mathrm{T}, a}\right)+W_{\mathrm{E}, a}\left(Q_{a} X_{\mathrm{s}}^{a}+W_{\mathrm{T}}\right)\right)+\mathcal{O}\left(c^{-2}\right) .
$$

The "gravito-electric" part $\Phi_{\mathrm{el}}^{a}$ of the influence of external bodies (independent of the velocity of the satellite) reads

$$
\begin{aligned}
\Phi_{\mathrm{el}}^{a}=Q_{a}+W_{\mathrm{T}, a}+\frac{1}{c^{2}} & \left(2(1+\gamma) W_{\mathrm{T}, T}^{a}-2(\beta+\gamma)\left(Q_{b} X_{\mathrm{s}}^{b}+W_{\mathrm{T}}\right)\left(Q_{a}+W_{\mathrm{T}, a}\right)\right. \\
& \left.+(1+\gamma) \varepsilon_{a b c} \dot{C}_{b} X_{\mathrm{s}}^{c}\right)+\mathcal{O}\left(c^{-4}\right),
\end{aligned}
$$


and the "gravito-magnetic" part $\Phi_{\mathrm{mg}}^{a}$ (depending on the velocity of the satellite) of the influence of external bodies is given by

$$
\begin{aligned}
\Phi_{\mathrm{mg}}^{a}= & -(1+2 \gamma)\left(W_{\mathrm{T}, T}+\dot{Q}_{b} X_{\mathrm{s}}^{b}\right) \dot{X}_{\mathrm{s}}^{a}-2(1+\gamma)\left(W_{\mathrm{T}, b}+Q_{b}\right) \dot{X}_{\mathrm{s}}^{b} \dot{X}_{\mathrm{s}}^{a} \\
& +\gamma\left(W_{\mathrm{T}, a}+Q_{a}\right) \dot{X}_{\mathrm{s}}^{b} \dot{X}_{\mathrm{s}}^{b}+4(1+\gamma) W_{\mathrm{T}}^{[a, b]} \dot{X}_{\mathrm{s}}^{b}+2(1+\gamma) \varepsilon_{a b c} C_{b} \dot{X}_{\mathrm{s}}^{c}+\mathcal{O}\left(c^{-2}\right),
\end{aligned}
$$

Let us remind that $\Phi_{\mathrm{el}}^{a}$ and $\Phi_{\mathrm{mg}}^{a}$ contain also the two arbitrary functions $Q_{a}$ and $C_{a}$ that characterize the world line of the local reference system and rotation of its spatial axes. A reasonable choice of $Q_{a}$ leading to vanishing of the $\mathrm{BD}$ mass dipole $\mathcal{M}^{a}$ of the central body will be discussed in Section IXE. As it was noted above (see the discussion after (4.12)) a dynamically nonrotating local reference system corresponds to $C_{a}=0$ while in a kinematically nonrotating local reference system $C_{a}$ contains the geodetic, Lense-Thirring and Thomas precessions.

The term $\Phi_{\Psi}^{a}$ represents the additional acceleration due to the term (4.25)

$$
\begin{aligned}
\Phi_{\Psi}^{a}(T, \mathbf{X}) & =\Psi_{, a}(T, \mathbf{X}) \\
& =-\eta\left(W_{\mathrm{E}, a}\left(\bar{w}\left(\mathbf{x}_{E}\right)+a_{E}^{i} r_{E}^{i}\right)-\chi_{, a b}^{\mathrm{E}}(T, \mathbf{X}) R_{i}^{b} a_{E}^{i}+W_{\mathrm{E}} R_{i}^{a} a_{E}^{i}\right)+\mathcal{O}\left(c^{-2}\right)
\end{aligned}
$$

In case of two spherically symmetrical nonrotating masses the term $\Psi$ (the potential of $\Phi_{\Psi}^{a}$ ) coincides with the 6th and 7th terms in $g_{00}$ of Eq. (52) in 64. A discussion of observability of this effect in satellite motion data can be found in Section VI of [64].

\section{B. Multipole expansions of the equations of motion}

For many applications it is convenient to expand the equations of motion derived in the previous Section into multipole moment of the gravitational field of the central body and into tidal moments of the external potentials. E.g., it is a common practice to use the equations of motion of near-Earth satellites in expanded form and to fit the multipole moments of the Earth's gravitational field. Substituting the expansions derived in Sections $\nabla \mathbb{1}$ and $\nabla 1]$ into (8.3)- (8.6) and (8.7) one gets 


$$
\begin{aligned}
& \Phi_{\mathrm{E}}^{a}=G \sum_{l=0}^{\infty} \frac{(-1)^{l}}{l !} \mathcal{M}_{L} \partial_{a L} \frac{1}{\left|\mathbf{X}_{\mathrm{s}}\right|} \\
& +\frac{1}{c^{2}} G \sum_{l=0}^{\infty} \frac{(-1)^{l}}{l !}\left(\frac{1}{2} \ddot{\mathcal{M}}_{L} \partial_{a L}\left|\mathbf{X}_{\mathrm{s}}\right|+\frac{2(1+\gamma)}{l+1} \ddot{\mathcal{M}}_{a L} \partial_{L} \frac{1}{\left|\mathbf{X}_{\mathrm{s}}\right|}-\frac{2(1+\gamma)}{l+2} \varepsilon_{a b c} \dot{\mathcal{S}}_{b L} \partial_{c L} \frac{1}{|\mathbf{X}|_{\mathrm{s}}}\right. \\
& -2(\gamma+\beta) \mathcal{M}_{L} \partial_{a L} \frac{1}{\left|\mathbf{X}_{\mathrm{s}}\right|}\left(G \sum_{k=0}^{\infty} \frac{(-1)^{k}}{k !} \mathcal{M}_{K} \partial_{K} \frac{1}{\left|\mathbf{X}_{\mathrm{s}}\right|}\right) \\
& +\gamma \dot{X}_{\mathrm{s}}^{b} \dot{X}_{\mathrm{s}}^{b} \mathcal{M}_{L} \partial_{a L} \frac{1}{\left|\mathbf{X}_{\mathrm{s}}\right|}-2(1+\gamma) \dot{X}_{\mathrm{s}}^{b} \dot{X}_{\mathrm{s}}^{a} \mathcal{M}_{L} \partial_{b L} \frac{1}{\left|\mathbf{X}_{\mathrm{s}}\right|} \\
& -(2 \gamma+1) \dot{X}_{\mathrm{s}}^{a} \dot{\mathcal{M}}_{L} \partial_{L} \frac{1}{\left|\mathbf{X}_{\mathrm{s}}\right|}+\frac{4(1+\gamma)}{l+1} \dot{X}_{\mathrm{s}}^{b} \dot{\mathcal{M}}_{L[a} \partial_{b] L} \frac{1}{\left|\mathbf{X}_{\mathrm{s}}\right|} \\
& \left.+\frac{4(1+\gamma)}{l+2} \dot{X}_{\mathrm{s}}^{b} \mathcal{S}_{c L} \varepsilon_{d c[a} \partial_{b] d L} \frac{1}{\left|\mathbf{X}_{\mathrm{s}}\right|}\right)+\mathcal{O}\left(c^{-4}\right) \\
& \Phi_{\text {coup }}^{a}=-2(\gamma+\beta) G\left(\sum_{l=0}^{\infty} \sum_{k=0}^{\infty} \frac{(-1)^{l}}{l !} \frac{1}{k !} \mathcal{M}_{L} Q_{a K} \hat{X}_{\mathrm{s}}^{K} \partial_{L} \frac{1}{\left|\mathbf{X}_{\mathrm{s}}\right|}\right. \\
& \left.+\sum_{l=0}^{\infty} \sum_{k=1}^{\infty} \frac{(-1)^{l}}{l !} \frac{1}{k !} \mathcal{M}_{L} Q_{K} \hat{X}_{\mathrm{s}}^{K} \partial_{a L} \frac{1}{\left|\mathbf{X}_{\mathrm{s}}\right|}\right)+\mathcal{O}\left(c^{-2}\right) \\
& \Phi_{\mathrm{el}}^{a}=\sum_{l=0}^{\infty} \frac{1}{l !} Q_{a L} \hat{X}_{\mathrm{s}}^{L}+\frac{1}{c^{2}}\left(2(1+\gamma) \sum_{l=1}^{\infty} \frac{1}{l !} \frac{l}{l+1} \varepsilon_{a b c} \dot{C}_{b L-1} \hat{X}_{\mathrm{s}}^{c L-1}\right. \\
& +\sum_{l=0}^{\infty} \frac{1}{l !} \frac{1}{2(2 l+3)} \ddot{Q}_{a L} \mathbf{X}_{\mathrm{s}}^{2} \hat{X}_{\mathrm{s}}^{L}-\sum_{l=2}^{\infty} \frac{1}{l !} \frac{(4 \gamma+3) l-2(1+\gamma)}{2 l+1} \ddot{Q}_{L-1} \hat{X}_{\mathrm{s}}^{a L-1} \\
& +\frac{1}{3} X_{\mathrm{s}}^{a}\left((\gamma-1) \ddot{\bar{U}}\left(\mathbf{x}_{E}\right)+2(1-\beta) a_{E}^{2}\right)-\frac{1}{6}(\gamma-1) R_{i}^{a} \ddot{a}_{E}^{i} \mathbf{X}_{\mathrm{s}}^{2} \\
& +\frac{2}{5}(\gamma-1) R_{i}^{b} \ddot{a}_{E}^{i} \hat{X}_{\mathrm{s}}^{a b}-\eta \sum_{l=0}^{\infty} \frac{1}{l !} \frac{1}{2 l+3} R_{i}^{b} a_{E}^{i} Q_{b L} \hat{X}_{\mathrm{s}}^{a L} \\
& -\frac{1}{2} \eta \sum_{l=0}^{\infty} \frac{1}{l !} \frac{1}{2 l+3} R_{i}^{b} a_{E}^{i} Q_{a b L} \mathbf{X}_{\mathrm{s}}^{2} \hat{X}_{\mathrm{s}}^{L} \\
& \left.-2(\gamma+\beta) \sum_{l=0}^{\infty} \sum_{k=1}^{\infty} \frac{1}{l !} \frac{1}{k !} Q_{a L} Q_{K} \hat{X}_{\mathrm{s}}^{L} \hat{X}_{\mathrm{s}}^{K}\right)+\mathcal{O}\left(c^{-4}\right), \\
& \Phi_{\mathrm{mg}}^{a}=-(2 \gamma+1) \dot{X}_{\mathrm{s}}^{a} \sum_{l=1}^{\infty} \frac{1}{l !} \dot{Q}_{L} \hat{X}_{\mathrm{s}}^{L}-2(1+\gamma) \dot{X}_{\mathrm{s}}^{a} \dot{X}_{\mathrm{s}}^{b} \sum_{l=0}^{\infty} \frac{1}{l !} Q_{b L} \hat{X}_{\mathrm{s}}^{L}+\gamma \dot{X}_{\mathrm{s}}^{b} \dot{X}_{\mathrm{s}}^{b} \sum_{l=0}^{\infty} \frac{1}{l !} Q_{a L} \hat{X}_{\mathrm{s}}^{L} \\
& -2(1+\gamma) \varepsilon_{a b c} \dot{X}_{\mathrm{s}}^{b} \sum_{l=0}^{\infty} \frac{1}{l !}\left(C_{c L} \hat{X}_{\mathrm{s}}^{L}+\frac{1}{l+2} \varepsilon_{c d e} \hat{X}_{\mathrm{s}}^{d L} \dot{Q}_{e L}\right) \\
& +(\gamma-1)\left(X_{\mathrm{s}}^{a} R_{i}^{b} \dot{a}_{E}^{i} \dot{X}_{\mathrm{s}}^{b}-R_{i}^{a} \dot{a}_{E}^{i} X_{\mathrm{s}}^{b} \dot{X}_{\mathrm{s}}^{b}\right)+\mathcal{O}\left(c^{-2}\right),
\end{aligned}
$$

where we use (7.15)-(7.16) and the identity $2 W_{\mathrm{T}}^{[a, b]}=\varepsilon_{a b c} \varepsilon_{c d e} W_{\mathrm{T}}^{d, e}$. 
The multipole expansion of $\Phi_{\Psi}^{a}$ reads

$$
\begin{aligned}
\Phi_{\Psi}^{a}=-\eta G \sum_{l=0}^{\infty} \frac{(-1)^{l}}{l !}\left\{\mathcal{M}_{L}(\right. & \left(\bar{w}\left(\mathbf{x}_{E}\right)+R^{b}{ }_{i} a_{E}^{i} X_{\mathrm{s}}^{b}\right) \partial_{a L} \frac{1}{\left|\mathbf{X}_{\mathrm{s}}\right|} \\
& \left.\quad+R^{a}{ }_{i} a_{E}^{i} \partial_{L} \frac{1}{\left|\mathbf{X}_{\mathrm{s}}\right|}-\frac{1}{2} R_{i}^{b} a_{E}^{i} \partial_{a b L}\left|\mathbf{X}_{\mathrm{s}}\right|\right) \\
& \left.-\frac{1}{2(2 l+3)} \mathcal{N}_{L} R_{i}^{b} a_{E}^{i} \partial_{a b L} \frac{1}{\left|\mathbf{X}_{\mathrm{s}}\right|}\right\}+\mathcal{O}\left(c^{-2}\right)
\end{aligned}
$$

where

$$
\mathcal{N}_{L}=\int_{V} \Sigma \mathbf{X}^{2} \hat{X}^{L} d^{3} X
$$

Here we use the multipole expansion of $\chi^{\mathrm{E}}(T, \mathbf{X})$

$$
\chi^{\mathrm{E}}(T, \mathbf{X})=\frac{1}{2} G \sum_{l=0}^{\infty} \frac{(-1)^{l}}{l !}\left(\mathcal{M}_{L} \partial_{L}|\mathbf{X}|+\frac{1}{2 l+3} \mathcal{N}_{L} \partial_{L} \frac{1}{|\mathbf{X}|}\right)+\mathcal{O}\left(c^{-2}\right),
$$

which can be verified directly from (4.24). The following relations are useful for evaluation of the expansions derived above

$$
\begin{aligned}
& \partial_{L} \frac{1}{|\mathbf{X}|}=(-1)^{l}(2 l-1) ! ! \frac{\hat{N}^{L}}{|\mathbf{X}|^{l+1}} \\
& \partial_{L}|\mathbf{X}|=(-1)^{l-1}(2 l-3) ! ! \frac{1}{|\mathbf{X}|^{l-1}}\left(\hat{N}^{L}-\frac{l(l-1)}{(2 l-1)(2 l-3)} \delta^{\left(a_{l-1} a_{l}\right.} \hat{N}^{L-2)}\right)
\end{aligned}
$$

where $N^{a}=X^{a} /|\mathbf{X}|$ and $\hat{N}^{L}=N^{\left\langle a_{1}\right.} N^{a_{2}} \ldots N^{\left.a_{l}\right\rangle}$. The shorthand notations $\partial_{L}\left(1 /\left|\mathbf{X}_{\mathrm{s}}\right|\right)$ and $\partial_{L}\left|\mathbf{X}_{\mathrm{s}}\right|$ mean $\partial_{L}(1 /|\mathbf{X}|)$ and $\partial_{L}|\mathbf{X}|$, correspondingly, evaluated at $\mathbf{X}=\mathbf{X}_{\mathrm{s}}$.

It is important to note that if $\eta \neq 0$ the equations of motion of a test particle contain not only the BD moments $\mathcal{M}_{L}$ and $\mathcal{S}_{L}$ as in case of General Relativity, but also one more family of mass moments $\mathcal{N}_{L}$ defined by (8.13).

\section{TRANSLATIONAL AND ROTATIONAL EQUATIONS OF MOTION OF $N$ EXTENDED BODIES}

Translational equations of motion of the origin of the local reference system is given by (4.28). In order to relate the origin of the local reference system to the post-Newtonian 
center of mass of the body (i.e., to fix $Q_{a}$ ) we can employ the local equations of motion in the local PPN reference system

$$
\mathcal{T}_{; \beta}^{\alpha \beta}=0
$$

\section{A. Local equations of motion: $\mathcal{T}_{; \beta}^{0 \beta}=0$}

Let us consider the integral

$$
\int_{V}(-\mathcal{G}) \mathcal{T}_{; \beta}^{0 \beta} d^{3} X=0
$$

In order to evaluate (9.2) we have to calculate the Christoffel symbols as well as the metric determinant for the local PPN metric

$$
\begin{aligned}
\Gamma_{00}^{0} & =-\frac{1}{c^{3}} W_{, t}+\mathcal{O}\left(c^{-5}\right), \\
\Gamma_{0 a}^{0} & =-\frac{1}{c^{2}} W_{, a}+\mathcal{O}\left(c^{-4}\right), \\
\Gamma_{a b}^{0} & =\frac{1}{c^{3}}\left((1+\gamma)\left(W_{, b}^{a}+W_{, a}^{b}\right)+\gamma \delta^{a b} W_{, T}\right)+\mathcal{O}\left(c^{-4}\right), \\
-\mathcal{G} & =1+\frac{1}{c^{2}} 2(3 \gamma-1) W+\mathcal{O}\left(c^{-4}\right) .
\end{aligned}
$$

Substituting (9.3)-(9.6) into (9.2) one gets

$$
\frac{d}{d T} M_{T}=F+\mathcal{O}\left(c^{-4}\right)
$$

where $M_{T}$ is the Tolman or ADM mass

$$
M_{T}(T)=\int_{V} \rho_{\mathrm{Tol}}(T, \mathbf{X}) d^{3} X,
$$

with

$$
\begin{aligned}
\rho_{\text {Tol }}(T, \mathbf{X})=\left(1+\frac{1}{c^{2}}\right. & \left(\left(\frac{1}{2}+3(\gamma-1)\right) W_{E}\right. \\
& \left.\left.+(3 \gamma-2)\left(Q_{a} X^{a}+W_{\mathrm{T}}\right)\right)\right) \frac{1}{c^{2}} \mathcal{T}^{00}+\mathcal{O}\left(c^{-4}\right) .
\end{aligned}
$$

In case of an ideal fluid 


$$
\rho_{\text {Tol }}(T, \mathbf{X})=\hat{\rho}^{*}\left(1+\frac{1}{c^{2}}\left(\frac{1}{2} V^{2}+\Pi-\frac{1}{2} W_{\mathrm{E}}\right)\right)+\mathcal{O}\left(c^{-4}\right),
$$

$\hat{\rho}^{*}$ being defined by (4.40). This justifies that $\rho_{\text {Tol }}$ as given by (9.9) is indeed the usual Tolman density. However, the definition (9.9) is independent of the model for $\mathcal{T}^{\mu \nu}$. The right-hand side of (9.7) reads

$$
F=\frac{1}{c^{2}} \int_{V} \Sigma^{a}\left(Q_{a}+W_{\mathrm{T}, a}\right) d^{3} X+\mathcal{O}\left(c^{-4}\right) .
$$

For an isolated body the mass $M_{T}$ is obviously conserved. The BD mass $\mathcal{M}$ defined by (6.1) is related with the Tolman mass $M_{T}$ by

$$
\begin{aligned}
\mathcal{M}=M_{T} & +\frac{1}{c^{2}} \eta \Omega_{\mathrm{E}}+\frac{1}{c^{2}} \frac{1}{6}(\gamma-1) \frac{d^{2}}{d T^{2}} \int_{V} \Sigma \mathbf{X}^{2} d^{3} X \\
& +\frac{1}{c^{2}}(1-\gamma-2 \beta) Q_{a} \int_{V} \Sigma X^{a} d^{3} X \\
& +\frac{1}{c^{2}} \int_{V} \Sigma\left((1-2 \beta) W_{\mathrm{T}}-\gamma W_{\mathrm{T}, a} X^{a}\right) d^{3} X+\mathcal{O}\left(c^{-4}\right),
\end{aligned}
$$

where

$$
\Omega_{\mathrm{E}}=-\frac{1}{2} \int_{V} \Sigma W_{\mathrm{E}} d^{3} X+\mathcal{O}\left(c^{-2}\right)
$$

is the internal gravitational energy of the body. One might have expected the appearance of the second term (containing $\Omega_{E}$ ) in (9.12) since $M_{T}$ is an inertial mass and $\mathcal{M}$ is the active gravitational mass appearing in the expansion of the gravitational potential (6.3) of the body. The third term can be related with a Newtonian virial theorem. It vanishes for an isolated body under the assumption of secular stationarity. The last two terms in (9.12) are proportional to $Q_{a}$ and the external tidal potential $W_{\mathrm{T}}$ respectively, and vanish for an isolated body. We see that the local equations of motion do not constrain the BD mass $\mathcal{M}$ as was the case in General Relativity, but only the Tolman mass $M_{T}$. Moreover, in the framework of the PPN formalism the $\mathrm{BD}$ mass $\mathcal{M}$ is not conserved even for an isolated body unless some specific physical conditions are met (secular stationarity and $\dot{\Omega}_{\mathrm{E}}=0$ ). 


\section{B. Local equations of motion: $\mathcal{T}_{; \beta}^{a \beta}=0$}

Let us now consider the integral

$$
\int_{V}(-\mathcal{G}) \hat{T}_{; \beta}^{a \beta} d^{3} X=0 .
$$

The Christoffel symbols necessary for the evaluation of (9.14) read

$$
\begin{aligned}
& \Gamma_{00}^{a}=-\frac{1}{c^{2}} W_{, a}+\frac{2}{c^{4}}\left((\beta+\gamma) W W_{, a}-(1+\gamma) W_{, T}^{a}\right)+\mathcal{O}\left(c^{-5}\right), \\
& \Gamma_{0 b}^{a}=\frac{1}{c^{3}}\left((1+\gamma)\left(W_{, a}^{b}-W_{, b}^{a}\right)+\gamma \delta^{a b} W_{, T}\right)+\mathcal{O}\left(c^{-5}\right), \\
& \Gamma_{b c}^{a}=\frac{1}{c^{2}} \gamma\left(-\delta^{b c} W_{, a}+\delta^{a b} W_{, c}+\delta^{a c} W_{, b}\right)+\mathcal{O}\left(c^{-4}\right),
\end{aligned}
$$

Substituting (9.15) $-(9.17)$ and (9.6) into (9.14) one gets

$$
\frac{d^{2}}{d T^{2}} M_{T}^{a}=F^{a}+\frac{1}{c^{2}} F_{\Psi}^{a}+\mathcal{O}\left(c^{-4}\right),
$$

where

$$
\begin{gathered}
M_{T}^{a}=\int_{V} \rho_{\mathrm{Tol}}(T, \mathbf{X}) X^{a} d^{3} X, \\
F^{a}=\int_{V} f^{a} d^{3} X+\frac{1}{c^{2}} \frac{d}{d T} \int_{V} g^{a} d^{3} X+\mathcal{O}\left(c^{-4}\right), \\
f^{a}=\Sigma\left(Q^{a}+W_{\mathrm{T}, a}\right)+\frac{1}{c^{2}} 2(1+\gamma)\left(\Sigma\left(\frac{1}{2} \varepsilon_{a b c} \dot{C}_{b} X^{c}+W_{\mathrm{T}, T}^{a}\right)\right. \\
\left.+\Sigma^{b}\left(-\varepsilon_{a b c} C_{c}+W_{\mathrm{T}, b}^{a}-W_{\mathrm{T}, a}^{b}\right)\right), \\
g^{a}=\Sigma^{b}\left(Q_{b}+W_{\mathrm{T}, \mathrm{b}}\right) X^{a}-(2 \gamma+1) \Sigma^{a}\left(Q_{b} X^{b}+W_{\mathrm{T}}\right), \\
F_{\Psi}^{a}=\int_{V} \Sigma \Psi_{, a} d^{3} X+\mathcal{O}\left(c^{-2}\right)=\eta \Omega_{\mathrm{E}} R_{i}^{a} a_{E}^{i}+\mathcal{O}\left(c^{-2}\right) .
\end{gathered}
$$

It is easy to see from (9.18)-(9.23) that for isolated body $\ddot{M}_{T}^{a}$ vanishes. The BD mass dipole $\mathcal{M}^{a}$ defined by (6.1) and $M_{T}^{a}$ are related by 


$$
\begin{aligned}
\mathcal{M}^{a}= & M_{T}^{a}+\frac{1}{c^{2}} \eta \Omega_{E}^{a} \\
& +\frac{1}{c^{2}} \frac{1}{5}(\gamma-1) \frac{d^{2}}{d T^{2}} \int_{V} \Sigma \mathbf{X}^{2} X^{a} d^{3} X-\frac{1}{c^{2}} \frac{1}{2}(\gamma-1) \frac{d}{d T} \int_{V} \Sigma^{a} \mathbf{X}^{2} d^{3} X \\
& +\frac{1}{c^{2}}(1-2 \beta) \int_{V} \Sigma\left(Q_{a} X^{a}+W_{\mathrm{T}}\right) X^{a} d^{3} X+ \\
& +\frac{1}{c^{2}} \frac{1}{2} \gamma \int_{V} \Sigma\left(Q_{a}+W_{\mathrm{T}, a}\right) \mathbf{X}^{2} d^{3} X \\
& -\frac{1}{c^{2}} \gamma \int_{V} \Sigma\left(Q_{b}+W_{\mathrm{T}, b}\right) X^{b} X^{a} d^{3} X+\mathcal{O}\left(c^{-4}\right),
\end{aligned}
$$

where

$$
\Omega_{\mathrm{E}}^{a}=-\frac{1}{2} \int_{V} \Sigma W_{\mathrm{E}} X^{a} d^{3} X+\mathcal{O}\left(c^{-2}\right) .
$$

If $\eta \neq 0$ or $\gamma \neq 1$, the second time derivative of the BD mass dipole $\ddot{\mathcal{M}}^{a}$ does not vanish even for one isolated body unless specific physical conditions are met (secular stationarity and $\left.\ddot{\Omega}_{\mathrm{E}}^{a}\right)$.

\section{Local equations of motion: $\varepsilon_{a b c} X^{b} \mathcal{T}_{; \beta}^{c \beta}=0$}

The local PPN reference system of an extended massive body described above allows us to derive also rotational equations of motion of the body in the framework of the PPN formalism. Substituting (9.15)-(9.17) and (9.6) into

$$
\varepsilon_{a b c} \int_{V}(-\mathcal{G}) X^{b} \mathcal{T}_{; \beta}^{c \beta} d^{3} X=0
$$

one gets the following rotational equations of motion (see, [28,70] for a more detailed discussion)

$$
\frac{d}{d T} S^{a}=L^{a}+\frac{1}{c^{2}} L_{\Psi}^{a}+\mathcal{O}\left(c^{-4}\right),
$$

where the PPN spin $S^{a}$ is defined by

$$
S^{a}=\varepsilon_{a b c} \int_{V} X^{b} p^{c} d^{3} X+\mathcal{O}\left(c^{-4}\right)
$$

with 


$$
\begin{gathered}
p^{a}=\Sigma^{a}\left(1+\frac{5 \gamma-1}{c^{2}} W\right)-\frac{1}{2 c^{2}} G \Sigma \int_{V} \Sigma^{b}\left(T, \mathbf{X}^{\prime}\right) \frac{(4 \gamma+3) \delta^{a b}+n^{a} n^{b}}{\left|\mathbf{X}-\mathbf{X}^{\prime}\right|} d^{3} X^{\prime}+\mathcal{O}\left(c^{-4}\right), \\
n^{a}=\frac{X^{a}-X^{\prime a}}{\left|\mathbf{X}-\mathbf{X}^{\prime}\right|} .
\end{gathered}
$$

Here,

$$
L^{a}=\varepsilon_{a b c} \int_{V} X^{b} f^{c} d^{3} X+\mathcal{O}\left(c^{-4}\right)
$$

and

$$
L_{\Psi}^{a}=\varepsilon_{a b c} \int_{V} \Sigma X^{b} \Psi_{, c} d^{3} X=\eta \varepsilon_{a b c} \Omega_{\mathrm{E}}^{b} R_{i}^{c} a_{E}^{i}+\mathcal{O}\left(c^{-4}\right),
$$

where $f^{a}$ and $\Omega_{\mathrm{E}}^{a}$ are defined by (9.21) and (9.25), respectively. For one isolated body $S^{a}$ is conserved. It is easy to see that $S^{a}$ coincide with the BD spin moment $\mathcal{S}^{a}$ defined by (6.2) in the Newtonian approximation

$$
S^{a}=\mathcal{S}^{a}+\mathcal{O}\left(c^{-2}\right)
$$

The torque $L_{\Psi}^{a}$ comes from the term $\Psi$ in the local PPN metric and represents an analogy of the Nordtvedt effect in the rotational equations of motion of an extended body in the local PPN metric. These rotational equations of motion and the torque $L_{\Psi}^{a}$ has been discussed in a previous paper [66].

\section{Multipole expansions of the relations between the Tolman and BD masses and mass dipoles}

For further analysis it is interesting to expand explicitly the relations for the Tolman mass $M_{T}$ and mass dipole $M_{T}^{a}$ with the BD mass $\mathcal{M}$ and mass dipole $\mathcal{M}^{a}$ defined by (9.12) and (9.24) respectively. One gets

$$
\begin{aligned}
\mathcal{M}=M_{T} & +\frac{1}{c^{2}} \eta \Omega_{E}+\frac{1}{c^{2}} \frac{1}{6}(\gamma-1) \ddot{\mathcal{N}} \\
& +\frac{1}{c^{2}} \sum_{l=1}^{\infty} \frac{1}{l !}(1-2 \beta-\gamma l) \mathcal{M}_{L} Q_{L}+\mathcal{O}\left(c^{-4}\right)
\end{aligned}
$$




$$
\begin{aligned}
\mathcal{M}^{a}= & M_{T}^{a}+\frac{1}{c^{2}} \eta \Omega_{\mathrm{E}}^{a} \\
& -\frac{1}{c^{2}} \frac{1}{10}(\gamma-1) \ddot{\mathcal{N}}_{a}+\frac{1}{c^{2}} \frac{3}{5}(\gamma-1) \dot{\mathcal{P}}_{a} \\
& +\frac{1}{c^{2}} \sum_{l=1}^{\infty} \frac{1}{l !}(1-2 \beta-\gamma l) \mathcal{M}_{a L} Q_{L}+ \\
& +\frac{1}{c^{2}} \sum_{l=0}^{\infty} \frac{1}{l !}\left(\frac{1}{2} \gamma-2 \beta+1\right) \frac{1}{2 l+3} \mathcal{N}_{L} Q_{a L}+\mathcal{O}\left(c^{-4}\right),
\end{aligned}
$$

where

$$
\mathcal{P}_{a}=\int_{V} \Sigma^{b} \hat{X}^{a b} d^{3} X
$$

Here, $\mathcal{N}_{L}$ is defined by 8.13$)$. The mass and mass dipole are the only moments which appear in two different forms in the formalism: 1) the BD mass and BD mass dipole in the multipole expansions of the internal gravitational potential (6.3), and 2) the Tolman mass and Tolman mass dipole in the left-hand side of the local equations of motions (9.7) and (9.18). The physical significance of the BD moments lies in their appearance in the

multipole expansion of the post-Newtonian gravitational field, that of the Tolman mass and mass dipole lies in representing integrals of local equations of motion for one isolated body. In General Relativity the BD and Tolman mass and mass dipoles coincide for isolated bodies. In contrast to this, within the PPN formalism this is no longer the case and the BD mass $\mathcal{M}$ and mass dipole $\mathcal{M}^{a}$ and the Tolman mass $M_{T}$ and mass dipole $M_{T}^{a}$ are essentially different objects. Higher multipole moments appear only in the multipole expansions of the internal gravitational potential (6.3).

\section{E. Multipole-expanded local translational equations of motion}

Using the definitions of the BD multipole moments and external tidal moments introduced in Sections $\mathrm{VI}$ and $\mathrm{VI}$ one can derive the following multipole expansions

$$
\begin{aligned}
\dot{\mathcal{M}}= & \Delta \dot{M} \\
& -\frac{1}{c^{2}} \sum_{l=1}^{\infty} \frac{1}{l !}\left((l+1+(\gamma-1) l+2(\beta-1)) \mathcal{M}_{L} \dot{Q}_{L}\right.
\end{aligned}
$$




$$
\left.+(l+(\gamma-1) l+2(\beta-1)) \dot{\mathcal{M}}_{L} Q_{L}\right)+\mathcal{O}\left(c^{-4}\right),
$$

$$
\begin{aligned}
& \ddot{\mathcal{M}}_{a}=\Delta \ddot{M}_{a}+R_{i}^{a} a_{E}^{i} \Delta M \\
& +\sum_{l=0}^{\infty} \frac{1}{l !} \mathcal{M}_{L} Q_{a L} \\
& -\frac{1}{c^{2}} 2(1+\gamma) \sum_{l=0}^{\infty} \frac{1}{l !}\left(\frac{1}{l+2} \mathcal{S}_{L+1} C_{a L+1}+\frac{1}{l+2} \varepsilon_{a b c} \mathcal{M}_{b L} \dot{C}_{c L}+\frac{1}{l+1} \varepsilon_{a b c} \dot{\mathcal{M}}_{b L} C_{c L}\right. \\
& \left.+\frac{(l+1)}{(l+2)^{2}} \varepsilon_{a b c} \mathcal{S}_{b L} \dot{Q}_{c L}+\frac{1}{l+2} \varepsilon_{a b c} \dot{\mathcal{S}}_{b L} Q_{c L}\right) \\
& -\frac{1}{c^{2}} \sum_{l=1}^{\infty} \frac{1}{l !}\left(\frac{(2 l+1)\left(l^{2}+3 l+6\right)}{(l+1)(2 l+3)}+(\gamma-1) \frac{2 l^{3}+5 l^{2}+7 l+2}{(l+1)(2 l+3)}+2(\beta-1)\right) \mathcal{M}_{a L} \ddot{Q}_{L} \\
& -\frac{1}{c^{2}} \sum_{l=1}^{\infty} \frac{1}{l !}\left(\frac{(2 l+1)\left(l^{2}+2 l+5\right)}{(l+1)^{2}}+2(\gamma-1) \frac{l^{3}+2 l^{2}+3 l+1}{(l+1)^{2}}+4(\beta-1)\right) \dot{\mathcal{M}}_{a L} \dot{Q}_{L} \\
& -\frac{1}{c^{2}} \sum_{l=1}^{\infty} \frac{1}{l !}\left(\frac{l^{2}+l+4}{l+1}+(\gamma-1) \frac{l^{2}+l+2}{l+1}+2(\beta-1)\right) \ddot{\mathcal{M}}_{a L} Q_{L} \\
& -\frac{1}{c^{2}} \eta \sum_{l=0}^{\infty} \frac{1}{l !} \frac{1}{2 l+3} \mathcal{M}_{a L} R_{i}^{b} a_{E}^{i} Q_{b L} \\
& -\frac{1}{2 c^{2}} \eta \sum_{l=0}^{\infty} \frac{1}{l !} \frac{1}{2 l+3} R_{i}^{b} a_{E}^{i} \mathcal{N}_{L} Q_{a b L} \\
& -\frac{1}{2 c^{2}} \eta \sum_{l=0}^{\infty} \frac{1}{l !} \frac{1}{2 l+3} \frac{d^{2}}{d T^{2}}\left(\mathcal{N}_{L} Q_{a L}\right) \\
& -\frac{1}{6 c^{2}}(\gamma-1) \frac{d^{2}}{d T^{2}}\left(\mathcal{N} R_{i}^{a}{ }_{i} a_{E}^{i}\right) \\
& +\frac{1}{3 c^{2}} \mathcal{M}_{a}\left((\gamma-1) \ddot{\bar{U}}\left(t, \mathbf{x}_{E}\right)-2(\beta-1) a_{E}^{2}\right)+\frac{2}{5 c^{2}}(\gamma-1) R_{i}^{b} \ddot{a}_{E}^{j} \mathcal{M}^{a b} \\
& +\frac{1}{c^{2}}(\gamma-1)\left(\frac{1}{2} \dot{\mathcal{M}}_{a b} R_{i}^{b} \dot{a}_{E}^{i}-\frac{1}{2} \varepsilon_{a b c} \mathcal{S}_{b} R_{i}^{c} \dot{a}_{E}^{i}\right)+\mathcal{O}\left(c^{-4}\right), \\
& \dot{\mathcal{S}}_{a}=\sum_{l=0}^{\infty} \frac{1}{l !} \varepsilon_{a b c} \mathcal{M}_{b L} Q_{c L}+\mathcal{O}\left(c^{-2}\right)
\end{aligned}
$$

with

$$
\Delta M=\frac{1}{c^{2}}\left(\eta \Omega_{E}+\frac{1}{6}(\gamma-1) \ddot{\mathcal{N}}\right)
$$

and

$$
\Delta M_{a}=\frac{1}{c^{2}}\left(\eta \Omega_{E}^{a}-\frac{1}{10}(\gamma-1) \ddot{\mathcal{N}}_{a}+\frac{3}{5}(\gamma-1) \dot{\mathcal{P}}_{a}\right)
$$


$\Delta M$ and $\Delta M_{a}$ represent the internal parts of the difference between the gravitational $\mathrm{BD}$ mass and mass dipole, $\mathcal{M}$ and $\mathcal{M}^{a}$, and the Tolman mass $M_{T}$ and $M_{T}^{a}$ given by (9.34)(9.35). In the limit of General Relativity (9.37)-(9.39) coincide with Eqs. (4.20a)-(4.21c) of [27].

Analogous to the translational equations of motion discussed in Section VIII, Eqs. (9.37)(9.38) contain not only the two families of BD moments $\mathcal{M}_{L}$ and $\mathcal{S}_{L}$, but one more family of multipole moments $\mathcal{N}_{L}$. Moreover, (9.37)-(9.38) contain also a number of additional terms

that vanish in General Relativity, but do not vanish for $\eta=0$. The physical meaning of some of these terms will be discussed in Section $\mathbb{\mathrm { XX }}$ below.

\section{F. Multipole-expanded rotational equations of motion}

In the same way for the post-Newtonian rotational equations of motion one gets

$$
\begin{array}{r}
\dot{S}^{a}=\varepsilon_{a b c} \sum_{l=0}^{\infty} \frac{1}{l !}\left(\mathcal{M}_{b L} Q_{c L}-\frac{1}{c^{2}} 2(1+\gamma) \frac{l+1}{l+2} \mathcal{S}_{b L} C_{c L}\right) \\
+\frac{1}{c^{2}} L_{\Delta}^{a}+\frac{1}{c^{2}} \frac{d}{d T} S_{1}^{a}+\frac{1}{c^{2}} \frac{d}{d T} S_{2}^{a}+\frac{1}{c^{2}} L_{\eta}^{a}+\mathcal{O}\left(c^{-4}\right)
\end{array}
$$

with

$$
\begin{aligned}
& L_{\Delta}^{a}=L_{\Psi}^{a}-\frac{1}{10}(\gamma-1) \varepsilon_{a b c} \ddot{\mathcal{N}}_{b} R_{i}^{c} a_{E}^{i}+\frac{3}{5}(\gamma-1) \varepsilon_{a b c} \dot{\mathcal{P}}_{b} R_{i}^{c} a_{E}^{i}=c^{2} \varepsilon_{a b c} \Delta M_{b} R_{i}^{c} a_{E}^{i}, \\
& S_{1}^{a}=-2(1+\gamma) \sum_{l=1}^{\infty} \frac{1}{l !} \frac{l}{l+1} \mathcal{M}_{a L} C_{L} \\
& S_{2}^{a}=2(1+\gamma) \sum_{l=0}^{\infty} \frac{1}{l !} \frac{1}{2 l+3} \mathcal{N}_{L} C_{a L} \\
&+2(1+\gamma) \varepsilon_{a b c} \sum_{l=0}^{\infty} \frac{1}{l !} \frac{(2 l+3)}{(l+2)(2 l+5)} \mathcal{P}_{b L} Q_{c L} \\
&+\varepsilon_{a b c} \sum_{l=0}^{\infty} \frac{1}{l !} \frac{l+2(\gamma+2)}{2(l+2)(2 l+5)}\left(\mathcal{N}_{b L} \dot{Q}_{c L}-\dot{\mathcal{N}}_{b L} Q_{c L}\right) \\
&+(1+\gamma) \varepsilon_{a b c} \sum_{l=0}^{\infty} \frac{1}{l !} \frac{1}{(l+2)(2 l+5)} \frac{d}{d T}\left(\mathcal{N}_{b L} Q_{c L}\right)
\end{aligned}
$$




$$
\begin{aligned}
& -\frac{3}{5}(\gamma-1) \varepsilon_{a b c} \mathcal{P}_{b} R_{i}^{c} a_{E}^{i} \\
& -\frac{1}{5}(\gamma-1) \varepsilon_{a b c}\left(\mathcal{N}_{b} R_{i}^{c} \dot{a}_{E}^{i}-\dot{\mathcal{N}}_{b} R_{i}^{c} a_{E}^{i}\right) \\
& -\frac{1}{10}(\gamma-1) \varepsilon_{a b c} \frac{d}{d T}\left(\mathcal{N}_{b} R_{i}^{c} a_{E}^{i}\right)
\end{aligned}
$$

and

$$
L_{\eta}^{a}=-\eta \varepsilon_{a b c} \sum_{l=0}^{\infty} \frac{1}{l !} \frac{1}{2(2 l+5)} \mathcal{N}_{b L} R_{i}^{d} a_{E}^{i} Q_{c d L}
$$

Here, $L_{\Psi}^{a}$ is defined by $(9.32)$ and $\mathcal{P}_{L}$ by (6.6). We prefer here to consider $L_{\Psi}^{a}$ as part of $L_{\Delta}^{a}$ which is proportional to the difference of the BD and Tolman dipoles $\Delta M_{a}$. This might provide a deeper insight into the nature of this torque. As in General Relativity we can now change the definition of the post-Newtonian spin into $S^{\prime a}=S^{a}+S_{1}^{a}+S_{2}^{a}$ to get rid of $S_{1}^{a}$ and $S_{2}^{a}$ on the right-hand side of (9.42) (see, [28] for a discussion of this point):

$$
\dot{S}^{\prime a}=\varepsilon_{a b c} \sum_{l=0}^{\infty} \frac{1}{l !}\left(\mathcal{M}_{b L} Q_{c L}-\frac{1}{c^{2}} 2(1+\gamma) \frac{l+1}{l+2} \mathcal{S}_{b L} C_{c L}\right)+\frac{1}{c^{2}}\left(L_{\Delta}^{a}+L_{\eta}^{a}\right)+\mathcal{O}\left(c^{-4}\right)
$$

The torque $L_{\eta}^{a}$ is in some sense similar to $L_{\Psi}^{a}$ : it is proportional to the Nordtvedt parameter $\eta$ and the acceleration of the body relative to the global reference system $a_{E}^{i}$. For that reason, it is related with a violation of the Strong Equivalence Principle. $L_{\Psi}^{a}$ has been discussed in detail in a previous paper [66].

Let us note here that the rotational equations of motion and the definition of the postNewtonian spin should be considered as being formal in the first place. Further analyses will be needed to see how these concepts can be used efficiently in practice.

\section{G. Mass monopole-spin dipole approximation}

Let us discuss the skeletonized equations of motion of $N$ extended massive bodies with full multipole structure derived above for the case that the $N$ bodies possess only mass and spin. The corresponding mass monopole-spin dipole model for the multipole structure of each body $B$ can be mathematically formulated as 


$$
\begin{aligned}
& \mathcal{M}_{L}=\mathcal{O}\left(c^{-4}\right), l \geq 1, \\
& \mathcal{S}_{L}=\mathcal{O}\left(c^{-2}\right), l \geq 2, \\
& \mathcal{N}_{L}=\mathcal{O}\left(c^{-2}\right), \quad l \geq 1, \\
& \mathcal{P}_{a}=\mathcal{O}\left(c^{-2}\right), \\
& \Omega^{a}=\mathcal{O}\left(c^{-2}\right) .
\end{aligned}
$$

These assumptions agree with what we expect from a naive Newtonian model of spherically symmetric rotating body. From (9.34)-(9.35), (9.37)-(9.38), (9.42)-(9.46) and (9.48)-(9.52) one finds

$$
\begin{gathered}
\dot{M}_{T}=\mathcal{O}\left(c^{-4}\right), \\
\mathcal{M}=M_{T}+\Delta M+\mathcal{O}\left(c^{-4}\right), \\
\mathcal{M}^{a}=M_{T}^{a}+\mathcal{O}\left(c^{-4}\right), \\
\Delta M R_{i}^{a} a_{E}^{i}+\mathcal{M} Q_{a}-\frac{1}{6 c^{2}} \eta \mathcal{N} R_{i}^{b} a_{E}^{i} Q_{a b}+\frac{1}{6 c^{2}}(1-\gamma) \frac{d^{2}}{d T^{2}}\left(\mathcal{N} R_{i}^{a} a_{E}^{i}\right) \\
-\frac{1}{c^{2}}(1+\gamma) \mathcal{S}_{b} C_{a b}-\frac{1}{c^{2}} \frac{1}{2}(\gamma-1) \varepsilon_{a b c} \mathcal{S}_{b} R_{i}^{c} \dot{a}_{E}^{i}=\mathcal{O}\left(c^{-4}\right) .
\end{gathered}
$$

Eq. (9.56) allows us to derive $Q_{a}$ for the case that the origin of the local reference system coincides with the $\mathrm{BD}$ center of mass of the central body $\left(\mathcal{M}_{a} \equiv 0\right)$. For convenience we split $Q_{a}$ into a part independent of the spin of the body $Q_{a}^{\mathrm{m}}$ (that is, $Q_{a}^{\mathrm{m}}$ corresponds to mass monopole model for the central body) and a part proportional to the spin of the body $Q_{a}^{\mathrm{s}}$

$$
\begin{aligned}
Q_{a}= & Q_{a}^{\mathrm{m}}+Q_{a}^{\mathrm{s}} \\
Q_{a}^{\mathrm{m}}= & -\frac{\Delta M}{\mathcal{M}} R_{i}^{a} a_{E}^{i}+\frac{1}{6 c^{2}} \eta \frac{\mathcal{N}}{\mathcal{M}} R_{i}^{b} a_{E}^{i} Q_{a b} \\
& -\frac{1}{6 c^{2}}(1-\gamma) \frac{1}{\mathcal{M}} \frac{d^{2}}{d T^{2}}\left(\mathcal{N} R_{i}^{a} a_{E}^{i}\right)+\mathcal{O}\left(c^{-4}\right) \\
Q_{a}^{\mathrm{s}}= & \frac{1}{c^{2}}(1+\gamma) \frac{1}{\mathcal{M}} \mathcal{S}_{b} C_{a b}-\frac{1}{2 c^{2}}(1-\gamma) \frac{1}{\mathcal{M}} \varepsilon_{a b c} \mathcal{S}_{b} R_{i}^{c} \dot{a}_{E}^{i}+\mathcal{O}\left(c^{-4}\right)
\end{aligned}
$$


From assumptions (9.48)-(9.49) and expansions (6.3)-(6.4) in the local PPN reference system of each body $B$ we have

$$
\begin{aligned}
& W_{B}=G \mathcal{M}_{B} \frac{1}{\left|\mathbf{X}_{B}\right|}+\mathcal{O}\left(c^{-4}\right), \\
& W_{B}^{a}=\frac{1}{2} G \varepsilon_{a b c} \mathcal{S}_{B}^{c} \partial_{b} \frac{1}{\left|\mathbf{X}_{B}\right|}+\mathcal{O}\left(c^{-2}\right) .
\end{aligned}
$$

Here, for each body the skeletonized-harmonic gauge (6.7) was used (see, [22] for more detail). $\mathbf{X}_{B}$ are spatial coordinates of the local PPN reference system of body $B$. Now we derive the potentials of body $B$ in the global PPN metric following the steps of Appendix $\mathrm{C}$ of 22. From (3.45), (4.6) and (4.7) one gets

$$
\frac{1}{\left|\mathbf{X}_{B}\right|}=\frac{1}{r_{B}}\left(1-\frac{1}{c^{2}}\left(\frac{1}{2}\left(\dot{x}_{B}^{i} n_{B}^{i}\right)^{2}+\gamma \bar{w}_{B}\left(\mathbf{x}_{B}\right)+\frac{1}{2} \gamma \ddot{x}_{B}^{i} r_{B}^{i}\right)\right)+\mathcal{O}\left(c^{-4}\right),
$$

where $r_{B}^{i}=x^{i}-x_{B}^{i}, n_{B}^{i}=r_{B}^{i} / r_{B}$. Here, $x_{B}^{i}$ are the coordinates of body $B$ in the global PPN reference system, and $\bar{w}_{B}$ is the external potential appearing, e.g., in (3.9) when constructing the local PPN reference system for body B. Then substituting (9.60)-(9.61) and (9.62) into (4.20) and (4.8) we get the explicit form of the gravitational potential of the body $B$ in the global PPN reference system

$$
\begin{aligned}
& w_{B}= \frac{G \mathcal{M}_{B}}{r_{B}}\left(1+\frac{1}{c^{2}}(\right. \\
&\left.+\gamma+\frac{1}{2}\right) \dot{x}_{B}^{2}-(2 \beta-1) \bar{w}_{B}\left(\mathbf{x}_{B}\right) \\
&\left.\left.+\frac{1}{2}\left(\dot{x}_{B}^{2}-\ddot{x}_{B}^{i} r_{B}^{i}-\left(\dot{x}_{B}^{i} n_{B}^{i}\right)^{2}\right)\right)\right) \\
&+\frac{1}{c^{2}}(1+\gamma) G \varepsilon_{i j k} \dot{x}_{B}^{i} s_{B}^{k} \partial_{j} \frac{1}{r_{B}}+\mathcal{O}\left(c^{-4}\right) .
\end{aligned}
$$

Here, $s_{B}^{i}=R_{i}^{a(B)} S_{B}^{a}$ and $R_{i}^{a(B)}$ is the rotational matrix $R_{i}^{a}$ appearing in the local PPN reference system for body $B$. The expression for the vector potential $W_{B}^{a}$ in the global reference system can be derived immediately from (4.8), 9.63) and (9.61)

$$
w_{B}^{i}=\frac{G \mathcal{M}_{B}}{r_{B}} \dot{x}_{B}^{i}+\frac{1}{2} G \varepsilon_{i j k} s_{B}^{k} \partial_{j} \frac{1}{r_{B}}+\mathcal{O}\left(c^{-2}\right) .
$$

Now we note that in analogy to (3.9) the gravitational potentials of the global PPN metric can be split into the sum of the contributions of the bodies 


$$
\begin{aligned}
& w=\sum_{B} w_{B}+\mathcal{O}\left(c^{-4}\right), \\
& w^{i}=\sum_{B} w_{B}^{i}+\mathcal{O}\left(c^{-2}\right) .
\end{aligned}
$$

Hence we get the explicit form of the gravitational potentials of the global PPN reference system within our mass monopole-spin dipole model (9.60)-(9.61)

$$
\begin{aligned}
w= & \sum_{B} \frac{G \mathcal{M}_{B}}{r_{B}}\left(1+\frac{1}{c^{2}}\left(\left(\gamma+\frac{1}{2}\right) \dot{x}_{B}^{2}-(2 \beta-1) \sum_{C \neq B} \frac{G \mathcal{M}_{C}}{r_{C B}}\right)\right) \\
& +\frac{1}{c^{2}} \frac{1}{2} \sum_{B} G \mathcal{M}_{B} r_{B, t t} \\
& +\frac{1}{c^{2}}(1+\gamma) \sum_{B} G \varepsilon_{i j k} \dot{x}_{B}^{i} s_{B}^{k} \partial_{j} \frac{1}{r_{B}}+\mathcal{O}\left(c^{-4}\right), \\
w^{i}= & \sum_{B} \frac{G \mathcal{M}_{B}}{r_{B}} \dot{x}_{B}^{i}+\frac{1}{2} \sum_{B} G \varepsilon_{i j k} s_{B}^{k} \partial_{j} \frac{1}{r_{B}}+\mathcal{O}\left(c^{-2}\right),
\end{aligned}
$$

where $\mathbf{r}_{C B}=\mathbf{x}_{C}-\mathbf{x}_{B}$ and $r_{C B}=\left|\mathbf{r}_{C B}\right|$ for any $C$ and $B$.

The equations of translational motion of body $E(4.28)$ can be now written as

$$
a_{E}^{i}=a_{E, \mathrm{~m}}^{i}+a_{E, \mathrm{~s}}^{i}
$$

Here the part $a_{E, \mathrm{~m}}^{i}$ depends only upon the masses of the bodies

$$
\begin{aligned}
a_{E, \mathrm{~m}}^{i}= & -\sum_{B \neq E} G \mathcal{M}_{B} \frac{r_{E B}^{i}}{r_{E B}^{3}}-R_{i}^{a} Q_{\mathrm{m}}^{a} \\
+ & \frac{1}{c^{2}} \sum_{B \neq E} G \mathcal{M}_{B} \frac{r_{E B}^{i}}{r_{E B}^{3}}\left\{(2 \beta-1) \sum_{C \neq B} \frac{G \mathcal{M}_{C}}{r_{B C}}+2(\gamma+\beta) \sum_{C \neq E} \frac{G \mathcal{M}_{C}}{r_{E C}}+2(1+\gamma) \frac{G \mathcal{M}_{E}}{r_{E B}}\right. \\
& +\frac{3}{2} \frac{\left(r_{E B}^{j} \dot{x}_{B}^{j}\right)^{2}}{r_{E B}^{2}}-\frac{1}{2} \sum_{C \neq E, B} G \mathcal{M}_{C} \frac{r_{E B}^{j} r_{B C}^{j}}{r_{B C}^{3}} \\
& \left.\quad-(1+\gamma) \dot{x}_{B}^{j} \dot{x}_{B}^{j}-\gamma \dot{x}_{E}^{j} \dot{x}_{E}^{j}+2(1+\gamma) \dot{x}_{E}^{j} \dot{x}_{B}^{j}\right\} \\
+ & \frac{1}{c^{2}} \sum_{B \neq E} G \mathcal{M}_{B} \frac{r_{E B}^{j}}{r_{E B}^{3}}\left\{2(1+\gamma) \dot{x}_{E}^{j}-(2 \gamma+1) \dot{x}_{B}^{j}\right\}\left(\dot{x}_{E}^{i}-\dot{x}_{B}^{i}\right) \\
- & \frac{1}{c^{2}}\left(2 \gamma+\frac{3}{2}\right) \sum_{B \neq E} \frac{G \mathcal{M}_{B}}{r_{E B}} \sum_{C \neq E, B} G \mathcal{M}_{C} \frac{r_{B C}^{i}}{r_{B C}^{3}}+\mathcal{O}\left(c^{-4}\right) .
\end{aligned}
$$

These are the PPN equations of motion for a system of mass monopoles. They agree with the well-known PPN Lorentz-Droste-Einstein-Infeld-Hoffmann equations of motion (see, e.g., [1]). Finally, $a_{E, \mathrm{~s}}^{i}$ are additional acceleration terms due to the spins of the bodies 


$$
\begin{aligned}
a_{E, \mathrm{~s}}^{i}= & -R_{i}^{a} Q_{\mathrm{s}}^{a} \\
& +\frac{1}{c^{2}}(1+\gamma) G \sum_{B \neq E}\left(\varepsilon_{i j k} s_{B}^{k}\left(\dot{x}_{E}^{l}-\dot{x}_{B}^{l}\right) \partial_{j l} \frac{1}{r_{E B}}+\varepsilon_{j l k} s_{B}^{k}\left(\dot{x}_{E}^{l}-\dot{x}_{B}^{l}\right) \partial_{j i} \frac{1}{r_{E B}}\right) \\
& +\mathcal{O}\left(c^{-4}\right) .
\end{aligned}
$$

The geodetic deviation $Q_{\mathrm{s}}^{a}$ due to spin of body $\mathrm{E}$ can be further simplified with the mass monopole-spin dipole model we consider. From (7.9), (9.66) and (9.67) one gets

$$
\begin{aligned}
R_{i}^{a} Q_{\mathrm{s}}^{a}= & -\frac{1}{c^{2}} \frac{1}{\mathcal{M}_{E}} \varepsilon_{i j k} s_{E}^{j} \dot{a}_{E}^{k} \\
& -\frac{1}{c^{2}}(1+\gamma) \frac{1}{\mathcal{M}_{E}} G \sum_{B \neq E} \mathcal{M}_{B}\left(\varepsilon_{j l k} s_{E}^{k}\left(\dot{x}_{E}^{l}-\dot{x}_{B}^{l}\right) \partial_{i j} \frac{1}{r_{E B}}\right. \\
& \left.+\varepsilon_{i j k} s_{E}^{k}\left(\dot{x}_{E}^{l}-\dot{x}_{B}^{l}\right) \partial_{j l} \frac{1}{r_{E B}}\right) \\
& +\frac{1}{2 c^{2}}(1+\gamma) \frac{1}{\mathcal{M}_{E}} G \sum_{B \neq E} s_{E}^{j} s_{B}^{k} \partial_{i j k} \frac{1}{r_{E B}}+\mathcal{O}\left(c^{-4}\right) .
\end{aligned}
$$

Here we used the identity $\varepsilon_{i j[k} A_{l] m}=\frac{1}{2} \varepsilon_{k l m} A_{i j}$ valid of any trace-free $A_{i j}$ (so that, $\left.A_{i i}=0\right)$. Note that

$$
\begin{aligned}
\partial_{i j} \frac{1}{r_{E B}} & \equiv \frac{3 r_{E B}^{i} r_{E B}^{j}-\delta^{i j} r_{E B}^{2}}{r_{E B}^{5}}, \\
\partial_{i j k} \frac{1}{r_{E B}} & \equiv \frac{3 r_{E B}^{2}\left(r_{E B}^{i} \delta^{j k}+r_{E B}^{j} \delta^{i k}+r_{E B}^{k} \delta^{i j}\right)-15 r_{E B}^{i} r_{E B}^{j} r_{E B}^{k}}{r_{E B}^{7}} .
\end{aligned}
$$

Equations (9.68)-(9.73) together with (9.58) for $\gamma=\beta=1$ agree with Eqs. (6.29)-(6.36) of [27]. These equations of motion can be derived from Lagrangian quoted in Section [. Note that we did not assume the constancy of the Blanchet-Damour masses $\mathcal{M}_{B}$. Strictly speaking one has to account for the time dependence of the masses $\mathcal{M}_{B}$ during a [numerical] integration of the equations of motion (9.69). It is also clear that this effect is very small and usually may be neglected in practical applications. Another point is that the Nordtvedt effect here is represented here not only by the terms $\eta \Omega_{B}$ and $\eta \Omega_{E}$, but through the whole difference of the BD active gravitational mass $\mathcal{M}$ and the inertial Tolman mass $M_{T}$ of each body. According to (9.54) and (9.40) the difference contains not only $\eta \Omega$, but also terms $(\gamma-1) \ddot{\mathcal{N}} / 6$ that do not vanish even for spherical bodies. 
Taking into account that the $\mathrm{BD}$ mass $\mathcal{M}_{B}$ for each body is the active gravitational mass (see, (9.12)), it is easy to show that these equations of motion coincide with Eqs. (6.31)(6.34) and (6.47) of [1] if only the two PPN parameters $\beta$ and $\gamma$ are retained there, all total derivatives of any internal integrals are dropped in our equations (secular stationarity is assumed in [1]) and the $\mathcal{N}$-dependent terms are neglected in our equations. The $\mathcal{N}$ dependent terms proportional to $\eta$ and to $\gamma-1$ can be explained by a coupling of the body's extension to the background Ricci tensor, as already mentioned in Section 四 and coincides with the corresponding terms derived by Nordtvedt in [71 73, 47].

The spin-dependent part of the acceleration (9.70)-(9.71) coincides with the known spinorbit coupling derived, e.g., in [60,61,53 and can be derived from Lagrangian (1.17). The first term in (9.71) is independent of the PPN parameters. It results from the well-known Thomas precession and has been first discussed by Damour [60] (see, also [61]).

For the mass monopole-spin dipole model (9.48)-(9.52) extended by assuming

$$
\mathcal{P}_{L}=\mathcal{O}\left(c^{-2}\right), \quad l \geq 2
$$

the rotational equations of motion (9.42) read

$$
\dot{S}^{a}=-\frac{1}{c^{2}}(1+\gamma) \varepsilon_{a b c} \mathcal{S}_{b} C_{c}+\frac{1}{c^{2}} \frac{2}{3}(1+\gamma) \frac{d}{d T}\left(N C_{a}\right)+\mathcal{O}\left(c^{-4}\right) .
$$

The second term in the right-hand side of (9.75) can be absorbed by changing the definition of the post-Newtonian spin as it was done in 28] for General Relativity.

If the local reference system is chosen to be kinematically nonrotating, $R_{i}^{a}=\delta_{i}^{a}$, then the first term in (9.75) gives precession of the spin due to geodetic, Lense-Thirring and Thomas precessions (recall that in a kinematically nonrotating local reference system $R_{i}^{a}=\delta^{a j}$ and $C_{a}$ represent just the rotation of the local reference system relative to the global one due to geodetic precession (see, (4.12)). If, however, a local dynamically nonrotating reference system is chosen then $C_{a}=0$ and the term vanishes. Thus, we prove that in the PPN formalism an extended massive body with mass and spin only undergoes the same relativistic precession in the background gravitational field as test massless gyroscope. This generalizes the corresponding result for General Relativity given in 28]. 


\section{OUTLOOK AND CONCLUSIONS}

In this paper we have formulated a new framework of relativistic celestial mechanics in the first post-Newtonian approximation with PPN parameters $\gamma$ and $\beta$. Here we list some of the most interesting results that were derived in the main part of the paper.

- It is impossible to construct a local reference system satisfying simultaneously proper-

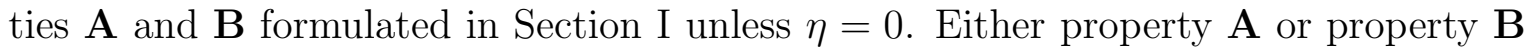
separately can be satisfied. For practical applications the local reference system where the external gravitational potential is represented by tidal effects only (i.e., property A is satisfied) seems to be more useful.

- As in General Relativity it is still possible to formulate the theory in terms of two material variables $\sigma$ and $\sigma^{i}$ related with $T^{\alpha \beta}$ by (3.5)-(3.6).

- It is possible to find an empirical definition of mass multipole moments (6.1)-(6.2) that allows us to keep the same almost Newtonian multipole expansion (6.3) of the local gravitational potential $W$ as in General Relativity. Those multipole moments generalize the Blanchet-Damour moments introduced in [69] in the framework of General Relativity.

- Unlike in General Relativity the BD-like mass and dipole moments (gravitational mass and mass dipole) do not coincide with the Tolman (or ADM) mass and dipole moment (inertial mass and mass dipole) even for one isolated body, where the BD-like mass in general is not conserved.

- The tidal expansions of external potentials in the local reference system involve $\beta$ and $\gamma$. All tidal terms can be divided into two groups: those which exist in General Relativity (coefficients now are polynomials of $\beta$ and $\gamma$ rather than rational numbers) and those vanishing in General Relativity. 
- Equations of motion of a test particle in the local reference system contain terms proportional to the acceleration of the central body relative to the global PPN reference system. The effect is proportional to $\eta$ and is a consequence of a violation of the Strong Equivalence Principle.

- For $\eta \neq 0$ the equations of motion of a test particle in the local reference system contain an additional family of multipole moments $\mathcal{N}_{L}$ defined by (8.13). Those moments also characterize the gravitational field of the central body, but do not appear in the multipole expansions (6.3)-(6.4) of post-Newtonian gravitational potentials $W$ and $W_{a}$

- For $\beta$ and $\gamma$ unequal to 1 equations of motion of both test particles (satellites) and massive bodies involve many additional terms proportional to $\eta, \gamma-1$ and $\beta-1$ which vanish in General Relativity. The terms proportional to $\eta$ come from a violation of the Strong Equivalence Principle, some other terms result from a coupling to the external Ricci tensor.

- It is no longer possible to construct a "point-mass" approximation to the equations of motion of $N$ extended bodies by assuming that all the bodies have constant BD mass and all other BD mass and spin multipole moments vanish identically as was the case in General Relativity [22]. A number of additional assumptions concerning the moments $\mathcal{N}_{L}$ as well as $\mathcal{P}_{a}$ and $\Omega_{a}$ is indispensable.

- If $\beta$ and $\gamma$ are unequal to 1 local equations of motions cannot be represented as a bilinear series of BD-like mass and spin multipole and tidal moments: one more family of multipole moments $\mathcal{N}_{L}$ and a number of additional terms (containing $\mathcal{P}_{a}, \Omega$ and $\left.\Omega^{a}\right)$ appear here.

- It is possible to derive equations of motion of $N$ massive extended bodies possessing only mass monopoles $\mathcal{M}$ (and moments of inertia $\mathcal{N}$ ), and, possibly, spin dipoles $\mathcal{S}^{i}$, without assuming secular stationarity. These equations of motion do not assume the 
gravitational masses of the bodies to be constant. The equations coincide with the known results (e.g., the EIH equations of motion) in the corresponding limits.

- Massive bodies possessing only mass monopoles $\mathcal{M}$ (and moments of inertia $\mathcal{N}$ ) no longer move along a geodesic of the background metric. This is usually called Nordtvedt effect and presents a direct consequence of a violation of the Strong Equivalence Principle. As compared with standard treatment of the Nordtvedt effect [1], the geodetic deviation of such a body contains not only terms $\eta \Omega$ proportional to internal gravitational energy of the bodies, but the complete differences between the gravitational and inertial masses of the bodies (9.40).

- Rotational equations of motion contain a term analogous to the Nordtvedt term in the translational equations of motion (i.e., a term proportional to $\eta$ and depending on the acceleration of the body relative to the global PPN metric). This question is discussed in [66] in more detail.

The framework presented here extends the Brumberg-Kopeikin and DSX-formalisms by the introduction of PPN-parameters. It also extends the classical Nordtvedt-Will PPNframework by: the formulation of a theory of astronomical reference frames with new transformation rules between global and local coordinates comoving with a body of the gravitational $N$-body system, improved definitions of mass and spin multipole moments that characterize the gravitational field of a body outside of its matter distribution in its own local coordinate system, and improved translational and rotational equations of motion for a system of $N$-extended rotating gravitationally interacting bodies of arbitrary shape and composition. This framework, in our opinion, shifts the 'pseudo Newtonian view' of the classical PPN-framework towards a more relativistic one. Note, that this point already was the motivation for the DSX-formalism. All main results from the classical PPN-framework as mentioned in the introduction (Section $\mathbb{~}$ ) are recovered, but now with the help of an improved and more consistent framework. Not only the usual PPN Einstein-Infeld-Hoffmann equations of motion for a system of mass monopoles, the spin-orbit and spin-spin interaction 
terms have been derived in the new $\beta, \gamma$-framework, but also all the various terms (depending upon $\eta,(\gamma-1)$ or $(\beta-1))$ related with a violation of the Strong Equivalence Principle are recovered.

It is the hope that this version of a PPN-formalism might eventually form the basis of a new test-theory for experimental gravity. To this end more work has to be done mainly concerning: a discussion of orders of magnitude of the various terms that appear in expansions in many places, the construction of models for the astronomical bodies, e.g., for the determination of the time dependence of the various mass and spin multipole moments of the bodies and, last not least, the relation of formalism with observations. This last point does not only concern the theoretical formulation of the various relevant measuring techniques (VLBI, SLR, LLR, GPS, astrometry, etc.) by employing the new PPN-framework, but the usefulness of certain theoretical concepts introduced here, e.g., the post-Newtonian spin of a body, has to be discussed. It is obvious that one wants to keep concepts that have been extremely useful in Newtonian classical mechanics for reasons of simplification also in a relativistic framework. However, the fact that many of these concepts do not even have a meaning in GRT (without resorting to some approximations) for real astronomical situations sheds some light on the difficulties that one faces here. On the other hand it is also obvious that the relevant measuring techniques at least in certain fields of application such as the problem of Earth's rotation have reached an accuracy level where a wealth of physical effects enters that simply cannot be modeled. In that case it has become a practice to start with a theoretical 'approximate model' and to measure 'offsets' from the theoretical predictions. What parts of the new PPN-framework presented here will eventually play a role in such an 'approximate model' (e.g., for the description of Earth's rotation) in order to reduce residuals is not so clear at present. 


\section{ACKNOWLEDGMENTS}

We are grateful to Prof. K. Nordtvedt for fruitful discussions. S.K. is also thankful to Dr. S.M. Kopeikin for a number of interesting discussions raising his interest to the problem of local reference systems in the PPN formalism. 


\section{REFERENCES}

[1] C.M. Will, Theory and Experiment in Gravitational Physics, Cambridge University Press, Cambridge (1993).

[2] M.H. Soffel, Relativity in Astrometry, Celestial Mechanics and Geodesy (Springer, Berlin, 1989).

[3] M. Froeschle, F. Mignard, F. Arenou, In: Proceedings of the ESA Symposium "Hipparcos - Venice 97", ESA SP-402, 49 (1997).

[4] S.A. Klioner, S.M. Kopeikin, Astron. J., 104, 897 (1992).

[5] L. Lindegren, M.A.C. Perryman, Astron. Astrophys. Supplement Series, 116, 579 (1996).

[6] Transactions of the International Astronomical Union, XXIB (edited by J.Bergeron), Dordrecht, Kluwer (1991).

[7] IERS Conventions, International Earth Rotation Service Technical Note 21 (ed. by D.D.McCarthy), Observatoire de Paris (1996).

[8] A. Einstein, Preuss. Akad. Wiss. Berlin, Sitzber., p.831, 18 November 1915

[9] J. Droste, Versl .K .Akad. Wet. Amsterdam, 19, 447 (1916).

[10] W. De Sitter, Mon. Not. R. Astr. Soc., 76, 699 (1916); 77, 155 (1916).

[11] H.A. Lorentz, J. Droste, Versl .K. Akad. Wet. Amsterdam, 26, 392 (part I) and 649 (part II) (1917). English translation in H.A. Lorentz, Collected Papers, ed. P. Zeeman, A,D, Fokker, vol. V, pp. 330-55. Martinus Nijhoff: The Hague (1937).

[12] V.A. Fock, Teoriya prostranstva vremeni i tyagoteniya (Fizmazgiz, Moscow, 1955); translated into English as The Theory of Space, Time and Gravitation, Pergamon, Oxford, (1959). 
[13] A. Papapetrou, Proc. R. Soc. London A 209, 248 (1951).

[14] S. Chandrasekhar, Astrophys. J., 142, 1488 (1965).

[15] S. Chandrasekhar, Y. Nutku, Astrophys. J., 158, 55 (1969).

[16] S. Chandrasekhar, F. Esposito, Astrophys. J., 160, 153 (1970).

[17] A. Caporali, Nuovo Cimento B 61, 181, 205 and 213 (1981).

[18] L.P. Grishchuk, S.M. Kopejkin, In: J. Kovalevsky, V.A. Brumberg (eds.), Relativity in Celestial Mechanics and Astrometry, Reidel, Dordrecht (1986).

[19] T. Damour, in: 300 Years of Gravitation, ed. S.W. Hawking, W. Israel, Cambridge University Press, Cambridge (1987).

[20] V.A. Brumberg, S.M. Kopejkin, in Reference Frames, edited by J. Kovalevsky, I.I. Mueller, B.Kolaczek, Kluwer, Dordrecht, 115 (1989).

[21] S.M. Kopejkin, Celestial Mechanics, 44, 87 (1988).

[22] T. Damour, M. Soffel, C. Xu, Phys. Rev. D 43, 3273 (1991).

[23] N. Ashby, B. Bertotti, Phys. Rev. Lett., 52, 485 (1984),

[24] N. Ashby, B. Bertotti, Phys. Rev., 34, 2246 (1986),

[25] V.A. Brumberg, S.M. Kopejkin, Nuovo Cimento, 103B, 63 (1989).

[26] S.M. Kopejkin, In: Itogi Nauki i Tekhniki, edited by M. V. Sazhin (Nauka, Moscow, 1991), 87 (in Russian).

[27] T. Damour, M. Soffel, C. Xu, Phys. Rev. D 45, 1017 (1992).

[28] T. Damour, M. Soffel, C. Xu, Phys. Rev. D 47, 3124 (1993).

[29] S.A. Klioner, A.V. Voinov, Phys. Rev. D, 48, 1451 (1993).

[30] J. Kovalevsky, I.I. Mueller, in Reference Coordinate Systems for Earth Dynamics, edited 
by E.M. Gaposchkin, B. Kolaczek, Reidel, Dordrecht, 375 (1981).

[31] P.K. Seidelmann, Celestial Mechanics, 37, 199 (1985).

[32] B. Guinot, Celestial Mechanics, 38, 155 (1986).

[33] T. Damour, M. Soffel, C. Xu, Phys. Rev. D 49, 618 (1994).

[34] S.M. Kopeikin, Soviet Astronomy, 34(1), 5 (1990).

[35] M. Soffel, X. Wu, Ch. Xu, J. Mueller, Astron. J., 101, 2306 (1991).

[36] S.A. Klioner, In: W.E. Carter (ed.), Proceedings of AGU Chapman Conference on Geodetic VLBI: Monitoring Global Change, NOAA Technical Report NOS 137 NGS 49, $188(1991)$.

[37] S.A. Klioner, Celestial Mechanics and Dynamical Astronomy, 53, 81 (1992).

[38] C.W. Misner, K.S. Thorne, J.A. Wheeler, Gravitation, Freeman, San Francisco, (1973)

[39] A.S. Eddington, The Mathematical Theory of Relativity, Cambridge University Press, Cambridge, England, (1922)

[40] H.P. Robertson, In: A.J. Deutsch, W.B. Klemperer (eds.), Space Age Astronomy, pp. 228-235, Academic Press, New York (1962).

[41] L.I. Schiff, in: Relativity Theory and Astrophysics. I. Relativity and Cosmology, ed. J. Ehlers, American Mathematical Society, Providence, (1967).

[42] K. Nordtvedt, Phys.Rev. 169, 1014 and 1017 (1968).

[43] K. Nordtvedt, Astrophys.J. 161, 1059 (1970).

[44] K. Nordtvedt, C. Will, Astrophys.J., 177, 775 (1972).

[45] C.M. Will, K. Nordtvedt, Astrophys.J., 177, 757 (1972).

[46] K. Nordtvedt, private communication (1998). 
[47] K. Nordtvedt, Phys. Rev. D49, 5165 (1994).

[48] M. Mathisson, Acta Phys. Polon., 6, 163 (1937).

[49] E. Corinaldesi, A. Papapetrou, Proc. Roy. Soc. London, 209A, 259 (1951).

[50] V.A. Brumberg, Relativistic Celestial Mechanics, Nauka, Moscow, (1972). (in Russian)

[51] N. Kalitzin, Nuovo Cimento 11, 178 (1959)

[52] R. Michalska, Bull. Acad. Polon. Sci., Ser. sci. math. astr. et phys. 8, 247 (1960).

[53] B.M. Barker, R.F. O’Connell, Phys. Rev. D12, 329 (1975),

[54] G. Börner, J. Ehlers, E. Rudolph, Astronomy and Astrophysics,44, 417 (1975).

[55] B.M. Barker, R.F. O’Connell, Phys. Rev., D14, 861 (1976),

[56] B.M. Barker, R.F. O'Connell, Phys. Rev., D24, 2332 (1981),

[57] B.M. Barker, G.G. Byrd, R.F. O’Connell, Astrophys. J., 253, 309 (1982),

[58] B.M. Barker, G.G. Byrd, R.F. O’Connell, Astrophys. J., 305, 623 (1986),

[59] W. Tulczyjew, Acta Phys. Pol., 18, 37 (1959).

[60] T. Damour, C. R. Acad. Sci. Paris, 294, série II, 1355 (1982).

[61] T. Damour, J.H. Taylor, Phys. Rev. D 45, 1840 (1992).

[62] Th. Apostolatos, Class. Quantum Grav., 13, 799 (1996).

[63] Th. Apostolatos, Phys. Rev. D 54, 2438 (1996),

[64] B. Shahid-Saless, N. Ashby, Phys. Rev. D38, 1645 (1988).

[65] S.A. Klioner, In: J. Vondrák, N. Capitaine (eds.), Reference systems and frames in the space era: present and future astrometric programmes, Proceeding of Les Journées 1997, Observatoire de Paris, (1997). 
[66] S.A. Klioner, M.H. Soffel, Phys. Rev. D, 58, 084023 (1998).

[67] S.A. Klioner, M.H. Soffel, Proc. of the 8th Marcel Grossmann Meeting, World Scientific, Singapore (1999) in press

[68] L. Blanchet, T. Damour, Phil. Trans. Roy. Soc. London, A320, 379 (1986).

[69] L. Blanchet, T. Damour, Ann. Inst. H. Poincaré, 50, 377 (1989).

[70] S.A. Klioner, In: S.Ferraz-Mello, B.Morando, J.E.Arlot (eds.), Dynamics, Ephemerides and Astrometry in the Solar System, Kluwer, Dordrecht, 309 (1996).

[71] K. Nordtvedt, Phys. Rev. D3, 1683 (1971).

[72] K. Nordtvedt, Astrophys. J., 254, 620 (1983).

[73] K. Nordtvedt, Phys. Rev. D43, 3131 (1991). 